INTER NATIONAL MONETARY FUND
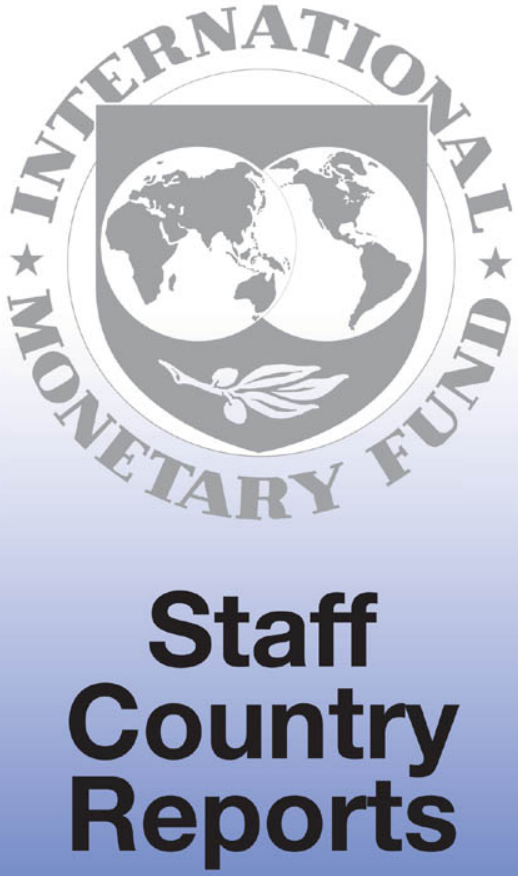
August 1998

IMF Staff Country Report No. 98/70

\section{Djibouti: Statistical Annex}

This Statistical Annex report on Djibouti was prepared by a staff team of the International Monetary Fund as background documentation for the periodic consultation with this member country. As such, the views expressed in this document are those of the staff team and do not necessarily reflect the views of the Government of Djibouti or the Executive Board of the IMF.

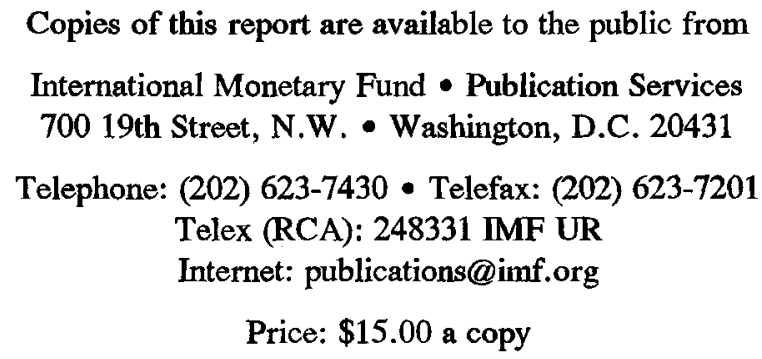

\section{International Monetary Fund \\ Washington, D.C.}




\section{INTERNATIONAL MONETARY FUND}

\section{DJIBOUTI}

\section{Statistical Annex}

Prepared by a staff mission consisting of

Messrs. Van-Can Thai, Edward Gardner, Montfort Mlachila, and Philippe Egoume-Bossogo

Approved by the Middle Eastern Department

May 22, 1998

Contents

Overview 4

\section{Tables}

\section{$\underline{\text { Real Sector }}$}

1. Basic Data, 1992-1997 . . . . . . . . . . . . . . . . . . . . . . . . . 5

2. Gross Domestic Product by Sector of Origin at

Current Prices, 1992-97

3. Gross Domestic Product by Sector of Origin at Current Prices, 1992-97, in percent of GDP 8

4. Supply and Use of Resources at Current Market Prices, 1992-97 9

5. Supply and Use of Resources at Current Market Prices, 1992-97, in percent of GDP

6. Agricultural Production by Species, 1992-1996 10

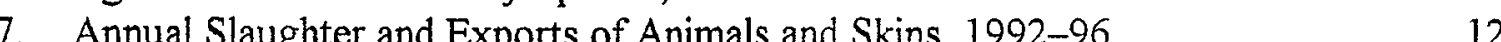

8. Purchases and Sales of Fish by the Maritime Cooperative, 1992-96 13

9. Production and Consumption of Electricity, 1992-97 14

10. Production and Consumption of Running Water in Urban Areas, 1992-96 15

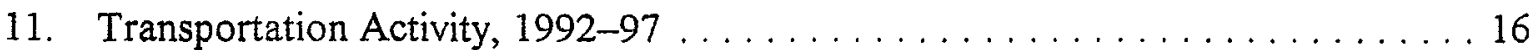

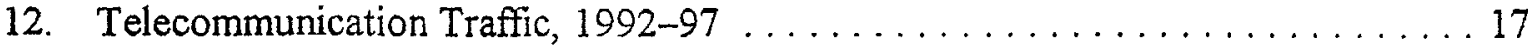


13. Postal and Telecommunication Tariffs in Force

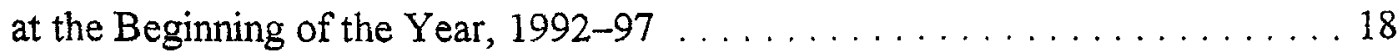

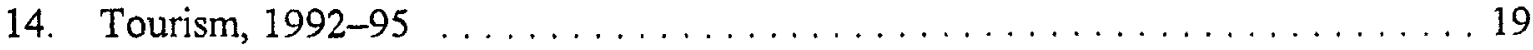

15. Expatriate Consumer Price Index by Product Group, $1992-97 \ldots \ldots \ldots \ldots 20$

16. Demand for Employment and Registered Offers at the National Employment Service, $1992-97$. . . . . . . . . . . . . . . 21

17. Number of Government Employees, $1992-97 \ldots \ldots \ldots \ldots \ldots \ldots \ldots . \ldots \ldots 22$

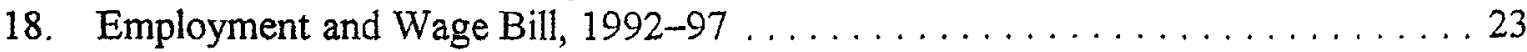

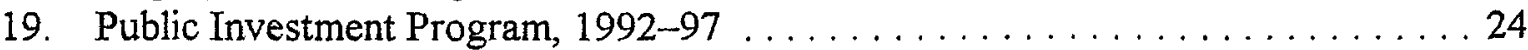

\section{$\underline{\text { Public Finance }}$}

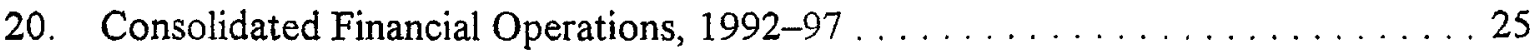

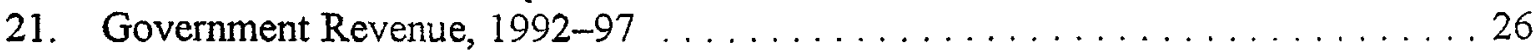

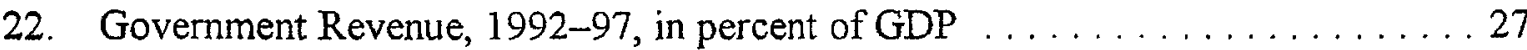

23. Government Revenue, $1992-97$, in percent of total revenue . . . . . . . . . 28

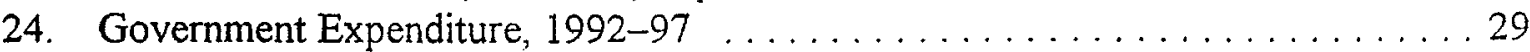

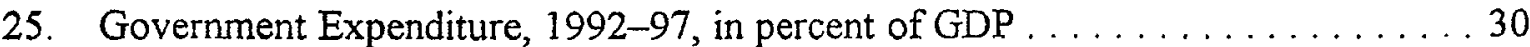

26. Government Expenditure, 1992-97, in percent of total expenditure . . . . . . . 31

27. Financing of Consolidated Fiscal Operations, $1992-97 \ldots \ldots \ldots \ldots \ldots \ldots 2$

28. Financing of Consolidated Fiscal Operations, 1992-97, in percent of total financing

29. Financing of Consolidated Fiscal Operations, 1992-97, in percent of GDP

\section{Monetary Sector}

30. Factors Affecting Changes in Domestic Liquidity, 1992-97 . . . . . . . . . . 35

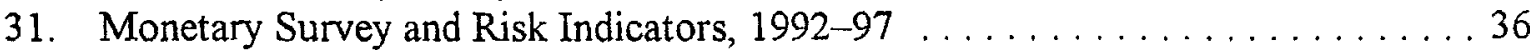

32. Analytical Accounts of the Monetary Authorities, $1992-97 \ldots \ldots \ldots$. . . . . . 37

33. Analytical Accounts of the Commercial Banks, $1992-97 \ldots \ldots \ldots \ldots . \ldots 38$

34. Analytical Accounts of the Development

Bank of Djibouti, 1992-97 . . . . . . . . . . . . . . . . . . . . . 39

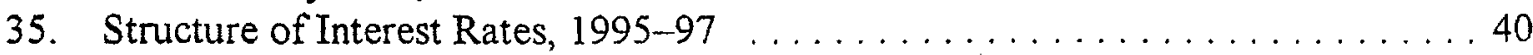

\section{External Sector}

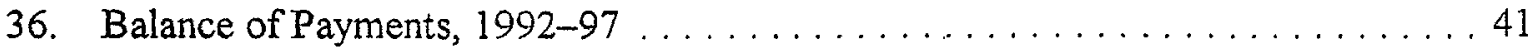

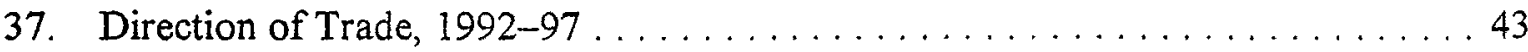

38. Domestically Consumed Imports Classified by Main Product Groups, 1992-97 
39. External Public- and Publicly-Guaranteed Debt, External Debt Service, 1993-97 45

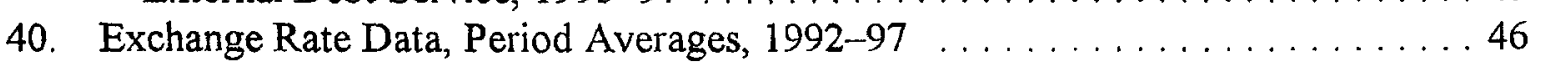

\section{Appendices}

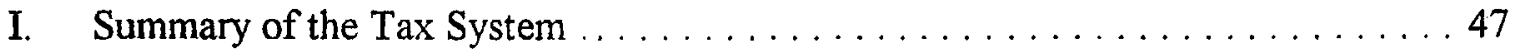

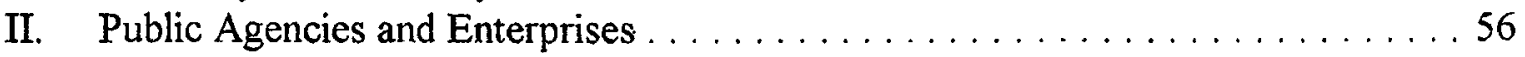




\section{Overview}

This document consists of two parts. First, it provides statistical information on the main economic sectors (real, fiscal, monetary, and external sectors). This information supplements the data contained in the staff report on the 1998 Article IV consultation, second review under the stand-by arrangement, and requests for extension, augmentation, and waiver of nonobservance of performance criteria (EBS/98/82, 05/18/98). Second, it also contains: (i) a summary of the tax system in Appendix I; and (ii) a list of public agencies and enterprises in Appendix II. 
Table 1. Djibouti: Basic Data, 1992-97

\begin{tabular}{llllllll}
\hline & 1992 & 1993 & 1994 & 1995 & $1996 \frac{1997}{\text { Prel. Est. }}$ \\
\hline
\end{tabular}

I. Economic and Financial Indicators

(Annual percentage changes)

National income and prices

GDP at current prices

GDP at constant prices

Consumer prices (annual average) I/

$3.1 \quad 0.3$

$\begin{array}{rr}-0.3 & -3.9\end{array}$

3.4

0.7

$-1.2$

3.1

Consolidated government financial operations $2 /$

\section{Revenue}

Grants

Current expenditure $3 /$

Investment expenditure $3 /$

Moniey and credit 4/

Net foreign assets

Net domestic assets

Claims on the central government (net)

Claims on the private sector $5 /$

Money and quasi money

External sector

Exports, f.o.b.

Imports, f.o.b.

Nominal effective exchange rate

(period averages; depreciation -)

Real effective exchange rate

(period averages; depreciation -)

Gross investment

Gross national savings

Government financial operations 3/

Total revenue and grants

Revenue

Grants

Total expenditure

Current expenditure

Capital expenditure

Overall deficit, payment order basis, excluding grants $6 /$

Overall deficit, payment order basis, excluding grants and demobilization

Overall deficit, cash basis, excluding grants $6 /$

Primary balance, excluding grants

Government payment arrears (stock)

\section{Domestic \\ External}

External sector

External current account (deficit $=-$ )

Excluding foreign budgetary transfers

$\begin{array}{rrrrrr}7.5 & 5.7 & 7.7 & -0.8 & 0.1 & -3.9 \\ 4.8 & -28.8 & -41.6 & -58.8 & 69.4 & 42.4 \\ 34.2 & 1.0 & -7.7 & -4.2 & -13.0 & 3.0 \\ 4.3 & -11.9 & -30.0 & -70.9 & 7.0 & 11.8\end{array}$

$\begin{array}{rrrrrr}-16.2 & 3.3 & -2.6 & -3.6 & -10.3 & -4.1 \\ 10.0 & -5.5 & 4.1 & 6.9 & 0.2 & 2.7 \\ 9.6 & 1.8 & 1.9 & 2.0 & -- & 2.5 \\ -0.8 & -3.8 & 1.1 & 8.0 & 2.0 & -0.3 \\ -6.2 & -2.2 & 1.5 & 3.3 & -10.0 & -1.4 \\ & & & & & \\ -25.7 & 33.9 & -20.9 & -33.3 & 5.3 & 7.6 \\ -- & -5.9 & -7.1 & -13.6 & -2.2 & 1.3 \\ -4.5 & 3.0 & 1.6 & -5.5 & 4.0 & 10.5 \\ -2.8 & 6.2 & 3.6 & -4.7 & 5.1 & 10.6\end{array}$

(In percent of GDP)

$\begin{array}{llllll}19.1 & 17.3 & 11.7 & 8.6 & 9.2 & 9.5\end{array}$

$\begin{array}{llllll}-2.8 & 4.8 & 1.4 & 2.0 & 2.6 & 3.1\end{array}$

$\begin{array}{llllll}39.3 & 37.2 & 34.5 & 31.1 & 31.3 & 30.0\end{array}$

$\begin{array}{llllll}26.9 & 28.4 & 29.5 & 29.1 & 29.5 & 27.5\end{array}$

$\begin{array}{lrrrrr}12.4 & 8.8 & 5.0 & 2.0 & 1.9 & 2.6\end{array}$

$\begin{array}{llllll}51.0 & 50.1 & 43.0 & 39.3 & 35.3 & 35.6\end{array}$

$\begin{array}{llllll}41.7 & 41.9 & 37.4 & 35.6 & 31.3 & 31.3\end{array}$

$\begin{array}{llllll}9.3 & 8.2 & 5.6 & 3.7 & 4.0 & 4.3\end{array}$

$\begin{array}{llllll}-24.1 & -21.8 & -13.4 & -10.1 & -5.8 & -8.1\end{array}$

$\begin{array}{llllll}-11.7 & -13.0 & -13.4 & -10.1 & -5.8 & -7.5\end{array}$

$\begin{array}{llllll}-23.7 & -18.0 & -11.1 & -7.0 & -6.5 & -7.1\end{array}$

$\begin{array}{llllll}-23.5 & -21.3 & -13.0 & -9.3 & -5.1 & -7.6\end{array}$

$\begin{array}{llllll}6.7 & 10.4 & 11.2 & 14.3 & 13.6 & 15.4\end{array}$

$\begin{array}{llllll}3.9 & 3.9 & 5.0 & 5.0 & 5.2 & 0.3\end{array}$

$\begin{array}{llllll}-28.3 & -15.1 & -13.2 & -5.9 & -5.9 & -6.4\end{array}$ 
Table 1. Djibouti: Basic Data, 1992-97

\begin{tabular}{|c|c|c|c|c|c|c|}
\hline & 1992 & 1993 & 1994 & 1995 & 1996 & $\frac{1997}{1 . \text { Est. }}$ \\
\hline & \multicolumn{6}{|c|}{ (In millions of U.S. dollars, unless otherwise indicated) } \\
\hline Overall balance of payments (deficit -) & -16.7 & -8.7 & 0.9 & -24.4 & 0.7 & -24.6 \\
\hline $\begin{array}{l}\text { Total external public debt } 7 / \\
\text { (in percent of GDP) }\end{array}$ & $\begin{array}{r}211.6 \\
45.0\end{array}$ & $\begin{array}{r}248.9 \\
52.8\end{array}$ & $\begin{array}{r}277.7 \\
57.0\end{array}$ & $\begin{array}{r}265.3 \\
54.1\end{array}$ & $\begin{array}{r}284.3 \\
58.6\end{array}$ & $\begin{array}{r}304.6 \\
60.9\end{array}$ \\
\hline $\begin{array}{l}\text { External debt service (in percent of } \\
\text { exports of locally produced goods } \\
\text { and nonfactor services) }\end{array}$ & 6.9 & 5.6 & 6.6 & 7.7 & 7.7 & 5.5 \\
\hline $\begin{array}{l}\text { Gross official reserves (in number of months } \\
\text { of imports of domestically consumed goods } \\
\text { and nonfactor services) }\end{array}$ & 3.5 & 3.3 & 3.7 & 3.5 & 3.8 & 3.4 \\
\hline $\begin{array}{l}\text { Exchange rate } \\
\text { Djibouti francs per U.S. dollar (end of period) }\end{array}$ & 177.7 & 177.7 & 177.7 & 177.7 & 177.7 & 177.7 \\
\hline
\end{tabular}

II. Social and Demographic Indicators
Area
23,200 sq km
Of which
0.4 percent arable land

Health

Life expectancy at birth (years) $8 /$

Infant mortality rate

(per thousand live births) 81

Population per physician (1991)

\section{Population}

660,000 (1996 estimate)

Rate of growth: 3 percent

Unemployment rate: 40 percent $8 /$

Under 20 years of age: 51 percent
Density

25.8 per sq. $\mathrm{km}$

\section{Education}

49.0 Gross enrollment ratio, primary (percent of school age children) 8/ secondary (percent of school age children) 81

Sources: Data provided by the Djibouti authorities; and Fund staff estimates.

1/ For expatriates only.

$2 /$ Fiscal year is the same as calendar year.

3/ Includes extrabudgetary outlays financed by external grants and loans.

4/ In relation to broad money stock at beginning of period.

$5 /$ Includes public enterprises.

6/ After taking into account the program of demobilization of security and military personnel.

7/ Public debt refers to the debt of both the central government and the public enterprise sector.

8/ Data refer to the latest figures available. 
Table 2. Djibouti: Gross Domestic Product by Sector of Origin at Current Prices, 1992-97

\begin{tabular}{|c|c|c|c|c|c|c|}
\hline & 1992 & 1993 & 1994 & 1995 & 1996 & $\frac{\text { Prel. }}{1997}$ \\
\hline & \multicolumn{6}{|c|}{ (In millions of Djibouti francs) } \\
\hline Primary sector & 2,189 & 2,327 & 2,397 & 2,517 & 2,673 & 2,860 \\
\hline Secondary sector & 15,346 & 15,193 & 14,768 & 14,842 & 15,346 & 16,101 \\
\hline Manufacturing & 3,944 & 3,924 & 3,872 & 4,105 & 4,311 & 4,462 \\
\hline Construction and public works & 4,275 & 4,318 & 4,189 & 4,440 & 6,035 & 6,639 \\
\hline Water and electricity & 7,127 & 6,950 & 6,707 & 6,297 & 5,000 & 5,000 \\
\hline Tertiary sector & 56,792 & 56,574 & 58,461 & 58,892 & 57,247 & 59,428 \\
\hline Commerce and tourism & 13,469 & 12,688 & 12,868 & 13,378 & 13,363 & 13,831 \\
\hline Transport and communications & 13,034 & 13,608 & 14,225 & 12,618 & 13,191 & 14,269 \\
\hline Barking and insurance $1 /$ & 7,528 & 7,091 & 7,192 & 7,928 & 8,277 & 8,566 \\
\hline Public administration & 19,838 & 20,135 & 20,997 & 21,107 & 18,364 & 18,547 \\
\hline Other services & 2,923 & 3,052 & 3,189 & 3,861 & 4,052 & 4,214 \\
\hline GDP at factor cost & 74,327 & 74,094 & 75,637 & 76,251 & 75,266 & 78,389 \\
\hline Net indirect taxes & 9,174 & 9,672 & 10,970 & 10,990 & 10,949 & 10,511 \\
\hline \multirow[t]{2}{*}{ GDP at market prices } & 83,501 & 83,766 & 86,607 & 87,241 & 86,215 & 88,900 \\
\hline & \multicolumn{6}{|c|}{ (Annual percentage change) } \\
\hline Primary sector & -2.7 & 6.3 & 3.0 & 5.0 & 6.9 & 7.0 \\
\hline Secondary sector & 13.9 & -1.0 & -2.8 & 0.5 & 3.4 & 4.9 \\
\hline Manufacturing & 10.5 & -0.5 & -1.3 & 6.0 & 3.1 & 3.5 \\
\hline Construction and public works & 12.0 & 1.0 & -3.0 & 6.0 & 35.7 & 10.0 \\
\hline Water and electricity & 17.1 & -2.5 & -3.5 & -6.1 & -4.0 & 0.0 \\
\hline Tertiary sector & 3.8 & -0.4 & 3.3 & 0.7 & -2.8 & 3.8 \\
\hline Commerce and tourism & -0.9 & -5.8 & 1.4 & 4.0 & 2.8 & 3.5 \\
\hline Transport and communications & -12.5 & 4.4 & 4.5 & -11.3 & -0.4 & 8.2 \\
\hline Banking and insurance $1 /$ & -0.9 & -5.8 & 1.4 & 10.3 & 4.0 & 3.5 \\
\hline Public administration & 29.9 & 1.5 & 4.3 & 0.5 & -5.0 & 1.0 \\
\hline Other services & -12.5 & 4.4 & 4.5 & 21.1 & 3.0 & 4.0 \\
\hline 3DP at factor cost & 5.5 & -0.3 & 2.1 & 0.8 & -0.1 & 4.1 \\
\hline Net indirect taxes & -13.0 & 5.4 & 13.4 & 0.2 & -7.3 & -4.0 \\
\hline JDP at market prices & 3.1 & 0.3 & 3.4 & 0.7 & -1.2 & 3.1 \\
\hline
\end{tabular}

Sources: Data provided by the Djibouti authorities; the World Bank; and Fund staff estimates.

1/ Net of imputed charge for banking services. 
Table 3. Djibouti: Gross Domestic Product by Sector of Origin at Current Prices 1007_07

(In percent of GDP)

$\begin{array}{llllll}1992 & 1993 & 1994 & 1995 & 1996 & \frac{\text { Prel. }}{1997}\end{array}$

$\begin{array}{lrrrrrr}\text { Primary sector } & 2.6 & 2.8 & 2.8 & 2.9 & 3.1 & 3.2 \\ & & & & & & \\ \text { Secondary sector } & 18.4 & 18.1 & 17.1 & 17.0 & 17.8 & 18.1 \\ \quad \text { Manufacturing and handicrafts } & 4.7 & 4.7 & 4.5 & 4.7 & 5.0 & 5.0 \\ \text { Construction and public works } & 5.1 & 5.2 & 4.8 & 5.1 & 7.0 & 7.5 \\ \text { Water and electricity } & 8.5 & 8.3 & 7.7 & 7.2 & 5.8 & 5.6 \\ & & & & & & \\ \text { Tertiary sector } & 68.0 & 67.5 & 67.5 & 67.5 & 66.4 & 66.8 \\ \text { Commerce and tourism } & 1.6 .1 & 15.1 & 14.9 & 15.3 & 15.5 & 15.6 \\ \text { Transport and communications } & 15.6 & 16.2 & 16.4 & 14.5 & 15.3 & 16.1 \\ \text { Banking and insurance } & 9.0 & 8.5 & 8.3 & 9.1 & 9.6 & 9.6 \\ \text { Public administration } & 23.8 & 24.0 & 24.2 & 24.2 & 21.3 & 20.9 \\ \text { Other services } & 3.5 & 3.6 & 3.7 & 4.4 . & 4.7 & 4.7 \\ & & & & & & \\ \text { GDP at factor cost } & 89.0 & 88.5 & 87.3 & 87.4 & 87.3 & 88.2 \\ \quad \text { Net indirect taxes } & 11.0 & 11.5 & 12.7 & 11.5 & 12.7 & 11.8\end{array}$

GDP at market prices

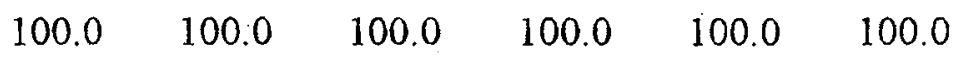

Sources: Data provided by the Djibouti authorities; the World Bank; and Fund staff estimates. 
Table 4. Djibouti: Supply and Use of Resources at Current Market Prices, 1992-97 (In millions of Djibouti francs)

\begin{tabular}{|c|c|c|c|c|c|c|}
\hline & 1992 & 1993 & 1994 & 1995 & 1996 & $\frac{\text { Prel. }}{1997}$ \\
\hline Supply of resources & 150,705 & 148,787 & 144,693 & 137,768 & 136,368 & 139,581 \\
\hline GDP at market prices & 83,501 & 83,765 & 86,607 & 87,241 & 86,215 & 88,900 \\
\hline \multicolumn{7}{|l|}{ Imports of goods and } \\
\hline Use of resources & 150,705 & 148,787 & 144,693 & 137,768 & 136,368 & 139,581 \\
\hline Total consumption & 99,015 & 92,944 & 96,386 & 94,795 & 92,822 & 94,448 \\
\hline Private & 62,073 & 56,040 & 61,625 & 61,400 & 63,943 & 69,609 \\
\hline Government & 36,942 & 36,904 & 34,761 & 33,395 & 28,879 & 24,839 \\
\hline Gross investment & 15,911 & 14,454 & 10,149 & 7,463 & 7,965 & 8,423 \\
\hline Private & 8,114 & 7,589 & 5,341 & 4,258 & 4,534 & 4,587 \\
\hline Government & 7,797 & 6,865 & 4,808 & 3,205 & 3,431 & 3,836 \\
\hline \multicolumn{7}{|l|}{ Exports of goods and } \\
\hline \multicolumn{7}{|l|}{ Memorandum items: } \\
\hline Gross national savings & $-2,328$ & 4,028 & 1,219 & 1,767 & 2,244 & 2,402 \\
\hline Resource gap & $-31,424$ & $-23,633$ & $-19,927$ & $-15,017$ & $-14,571$ & $-13,972$ \\
\hline
\end{tabular}

Sources: Data provided by Djibouti authorities; the World Bank; and Fund staff estimates. 
Table 5. Djibouti: Supply and Use of Resources at Current Market prices, 1992-97 (In vercent of GDP)

\begin{tabular}{|c|c|c|c|c|c|c|}
\hline & 1992 & 1993 & 1994 & 1995 & 1996 & $\frac{\text { Prel. }}{1997}$ \\
\hline Supply of resources & 180.5 & 177.6 & 167.1 & 157.9 & 158.2 & 157.0 \\
\hline GDP at market prices & 100.0 & 100.0 & 100.0 & 100.0 & 100.0 & 100.0 \\
\hline $\begin{array}{l}\text { Imports of goods and nor } \\
\text { services }\end{array}$ & 80.5 & 77.6 & 67.1 & 57.9 & 58.2 & 57.0 \\
\hline Use of resources & 180.5 & 177.6 & 167.1 & 157.9 & 158.2 & 157.0 \\
\hline Total consumption & 118.6 & 111.0 & 111.3 & 108.7 & 107.7 & 106.2 \\
\hline Private & 74.3 & 66.9 & 71.2 & 70.4 & 74.2 & 78.3 \\
\hline Government & 44.2 & 44.1 & 40.1 & 38.3 & 33.5 & 27.9 \\
\hline Gross investment & 19.1 & 17.3 & 11.7 & 8.6 & 9.2 & 9.5 \\
\hline Private & 9.7 & 9.1 & 6.2 & 4.9 & 5.3 & 5.2 \\
\hline Government & 9.3 & 8.2 & 5.6 & 3.7 & 4.0 & 4.3 \\
\hline \multicolumn{7}{|c|}{ Exports of goods and nonfactor } \\
\hline \multicolumn{7}{|l|}{ Memorandum items: } \\
\hline Gross national savings & -2.8 & 4.8 & 1.4 & 2.0 & 2.6 & 2.7 \\
\hline Resource gap & -37.6 & -28.2 & -23.0 & -17.2 & -16.9 & -15.7 \\
\hline
\end{tabular}

Sources: Data provided by the Djibouti authorities; the World Bank; and Fund staff estimates. 
Table 6. Djibouti: Agricultural Production by Species, 1992-96

(In tons)

\begin{tabular}{|c|c|c|c|c|c|}
\hline & 1992 & 1993 & 1994 & 1995 & $19961 /$ \\
\hline Eggplant & 33 & 22 & 39 & 39 & 43 \\
\hline Beetroot & 19 & 13 & 3 & 6 & 7 \\
\hline Carrot & 3 & 2 & 3 & 3 & 3 \\
\hline Cauliflower/Cabbage & 9 & 6 & 17 & 9 & 10 \\
\hline Cucumber & 5 & 2 & $\cdots$ & .. & $\ldots$ \\
\hline Zucchini & 5 & 2 & $\ldots$ & $\ldots$ & ... \\
\hline Bush beans & 5 & 6 & $\ldots$ & $\ldots$ & $\ldots$ \\
\hline Corn and dourah & 5 & 3 & $\ldots$ & $\ldots$ & $\ldots$ \\
\hline Melon & 103 & 67 & 187 & 82 & 91 \\
\hline Onion & 65 & 42 & 11 & 19 & 21 \\
\hline Watermelon & 43 & 28 & 78 & 65 & 72 \\
\hline Red pepper & 167 & 109 & 158 & 274 & 304 \\
\hline Leek & 100 & -- & .. & $\ldots$ & $\ldots$ \\
\hline Oranges & 454 & 856 & 867 & 438 & 486 \\
\hline Lemons & 906 & 1,087 & 1,304 & 1,565 & 1,733 \\
\hline Dates & 136 & 257 & 260 & 132 & 146 \\
\hline Mangoes & 327 & 392 & 471 & 565 & 626 \\
\hline Other (including fruits) & 607 & 572 & 542 & 1,216 & 1,346 \\
\hline
\end{tabular}

Source: Data provided by the Djibouti authorities.

1/ Latest data available. 
Table 7. Djibouti: Annual Slaughter and Exports of Animals and Skins, 1992-96

\begin{tabular}{lrrrrr}
\hline & 1992 & 1993 & 1994 & 1995 & $1996 \mathrm{I} /$ \\
\hline & & & & & \\
Number of animals & & & & & \\
$\quad$ Slaughtered & 229,024 & 180,317 & 138,970 & 138,002 & 142,541 \\
$\quad$ Sheep and goats & 196,803 & 145,030 & 114,247 & 113,846 & 118,128 \\
$\quad$ Cattle & 32,022 & 35,148 & 24,608 & 24,113 & 24,376 \\
$\quad$ Camels & 113 & 113 & 115 & 43 & 37 \\
Export of animals & & & & & \\
$\quad$ on the hoof & 4,000 & 1,100 & 2,825 & 2,825 & 2,826 \\
$\quad$ Sheep and goats & -- &.- & 825 & 825 & 825 \\
Cattle & 4,000 & 1,100 & 2,000 & 2,000 & 2,001 \\
& & & & & \\
& 395 & 385 & 323 & & $\ldots$ \\
Exports of skins & & & & & \\
\hline
\end{tabular}

Source: Data provided by the Djibouti authorities.

1/ Latest data available. 
Table 8. Djibouti: Purchases and Sales of Fish by the Maritime Cooperative, 1992-96 (Kilograms)

\begin{tabular}{lrrrrr}
\hline & 1992 & 1993 & 1994 & 1995 & $19961 /$ \\
\hline $\begin{array}{l}\text { Purchases } \\
\text { Lobsters } \\
\text { Fish, mollusks, and } \\
\quad \text { other shellfish }\end{array}$ & 125,700 & 55,917 & 310,877 & 335,747 & 361,230 \\
& 125,700 & 55,803 & 310,877 & 335,747 & 361,015 \\
$\begin{array}{l}\text { Sales } \\
\text { Individuals }\end{array}$ & $\ldots$ & 55,917 & 310,877 & $\ldots$ & $\ldots$ \\
$\quad$ Fish stores & $\ldots$ & $\ldots$ & $\ldots$ & $\ldots$ & $\ldots$ \\
Exports & $\ldots$ & $\ldots$ & $\ldots$ & $\ldots$ & $\ldots$ \\
\hline
\end{tabular}

Source: Data provided by the Djibouti authorities.

1/ Latest data available. 
Table 9. Djibouti: Production and Consumption of Electricity, 1992-97

\begin{tabular}{|c|c|c|c|c|c|c|}
\hline & 1992 & 1993 & 1994 & 1995 & 1996 & 1997 \\
\hline Production & 212,698 & 220,339 & 224,085 & 225,608 & 202,494 & 200,717 \\
\hline Number of users & 28,826 & 29,878 & 30,732 & 32,537 & 27,723 & 28,599 \\
\hline Consumption & 198,695 & 206,341 & 209,506 & 212,072 & 167,002 & 171,875 \\
\hline Industry & 95,737 & 99,421 & 117,180 & 118,761 & 78,391 & 79,168 \\
\hline Households & 102,958 & 106,920 & 92,326 & 93,311 & 88,611 & 92,707 \\
\hline Sales & 6,318 & 6,561 & 6,837 & 7,198 & 6,592 & 6,550 \\
\hline Industry & 3,045 & 3,161 & 3,933 & 3,407 & 3,120 & 3,118 \\
\hline Households & 3,273 & 3,400 & 2,904 & 3,791 & 3,472 & 3,432 \\
\hline Average electricity price & 37.0 & 37.0 & 37.0 & 37.0 & 39.0 & 38.0 \\
\hline
\end{tabular}

Source: Data provided by the Djibouti authorities. 
Table 10. Djibouti: Production and Consumption of Running Water in Urban Areas, 1992-96

(In cubic meters)

\begin{tabular}{lrrrrr}
\hline & 1992 & 1993 & 1994 & 1995 & $19961 /$ \\
\hline Production & 13,855 & 13,350 & 14,908 & 13,750 & 14,049 \\
Consumption & 9,865 & 9,237 & 9,976 & 9,727 & 9,525 \\
\hline
\end{tabular}

Source: Data provided by the Djibouti authorities.

1/ Latest data available. 
Table 11. Djibouti: Transportation Activity, 1992-97

\begin{tabular}{|c|c|c|c|c|c|c|}
\hline & 1992 & 1993 & 1994 & 1995 & 1996 & 1997 \\
\hline \multicolumn{7}{|l|}{ Seaport traffic } \\
\hline Passenger (number) & 6,103 & 7,262 & 11,746 & 5,317 & 7,256 & 3,812 \\
\hline Total merchandise ( 1,000 tons) $1 /$ & 1,302 & 1,018 & 1,207 & 736 & 756 & 720 \\
\hline Loaded and unloaded & 916 & 695 & 844 & 530 & 451 & 508 \\
\hline $\begin{array}{l}\text { Oil unloaded, bunkered, } \\
\text { re-exported }\end{array}$ & 386 & 323 & 363 & 206 & 304 & 212 \\
\hline $\begin{array}{l}\text { Water supplied to ships, } \\
\text { in cubic meters }\end{array}$ & 70 & 53 & 33 & 51 & 44 & 44 \\
\hline \multicolumn{7}{|l|}{ Airport traffic } \\
\hline \multicolumn{7}{|l|}{ Arrivals and departures } \\
\hline Number of airplanes & 5,392 & 7,380 & 9,906 & 9,036 & 8,402 & 8,382 \\
\hline Passengers (number) $2 /$ & 122,414 & 136,668 & 130,996 & 120,145 & 109,923 & 106,823 \\
\hline Freight (in tons) & 15,245 & 10,744 & 9,645 & 12,291 & 8,253 & 9,480 \\
\hline Postal items (in tons) & 450 & 463 & 383 & 338 & 337 & 336 \\
\hline \multicolumn{7}{|l|}{ Overall air traffic } \\
\hline Number of traffic units $3 /$ & 279 & 246 & 232 & 243 & $\ldots$ & $\ldots$ \\
\hline Percentage change & -47 & -12 & -6 & 5 & $\ldots$ & $\ldots$ \\
\hline \multicolumn{7}{|l|}{ Train traffic } \\
\hline $\begin{array}{l}\text { Passengers/kilometer } \\
\text { Merchandise (millions of tons/ }\end{array}$ & $\ldots$ & $\ldots$ & 592 & 279 & $\ldots$ & $\ldots$ \\
\hline kilometer) & 137 & 152 & 112 & 273 & $\ldots$ & $\ldots$ \\
\hline Percentage change & 5 & 11.4 & -26 & 143 & & \\
\hline \multicolumn{7}{|l|}{ Road vehicles } \\
\hline Net new registration 4/ & 1,229 & 1,067 & 846 & 1,200 & $\ldots$ & $\ldots$ \\
\hline $\begin{array}{l}\text { Percentage change } \\
\text { Of which }\end{array}$ & $\ldots$ & $\ldots$ & $\ldots$ & $\ldots$ & $\ldots$ & $\ldots$ \\
\hline $\begin{array}{l}\text { four-wheel vehicles } \\
\text { (gross) }\end{array}$ & $\cdots$ & $\cdots$ & 823 & $\ldots$ & $\ldots$ & . \\
\hline \multicolumn{7}{|l|}{ Motor vehicle annual } \\
\hline licenses sold & 7,051 & 6,820 & 6,578 & $\ldots$ & $\ldots$ & $\ldots$ \\
\hline
\end{tabular}

Source: Data provided by the Djibouti authorities.

1/ Sum of loaded and unloaded merchandise, and oil traffic.

2/ Sum of passengers arriving and departing, excluding those in transit (including military planes).

3 / One unit of traffic equals 1,000 passengers equals 100 tons of freight equals 100 tons of postal traffic. 4/ Includes net registration of all new four-wheel velicles plus motorcycles less changes of registration numbers. 
Table 12. Djibouti: Telecommunication Traffic, 1992-97

\begin{tabular}{|c|c|c|c|c|c|c|c|c|c|c|}
\hline & \multirow{3}{*}{$\begin{array}{r}\text { Telephone } \\
\text { Number } \\
\text { of } \\
\text { Users }\end{array}$} & \multirow{2}{*}{\multicolumn{2}{|c|}{$\begin{array}{c}\text { International } \\
\text { ('000 minutes) }\end{array}$}} & \multirow{3}{*}{$\begin{array}{r}\text { National } \\
\text { ('000 of Com- } \\
\text { munications) }\end{array}$} & \multirow{3}{*}{$\begin{array}{r}\frac{\text { Facsimile }}{\text { Number }} \\
\text { of } \\
\text { Users }\end{array}$} & \multicolumn{2}{|c|}{ Domestic } & \multicolumn{3}{|c|}{ International } \\
\hline & & & & & & Sent & Received & Sent & Received & In Transit \\
\hline & & Sent & Received & & & & & & & \\
\hline 1992 & 6,339 & 3,242 & $\ldots$ & $\ldots$ & 193 & $\ldots$ & 559 & 9,024 & 3,682 & 58 \\
\hline 1993 & 7,349 & 4,228 & $\ldots$ & $\ldots$ & 171 & $\ldots$ & 1,449 & 8,701 & 2,785 & $\ldots$ \\
\hline 1994 & 7,436 & 4,578 & $\ldots$ & 25,300 & 155 & $\ldots$ & 110 & 4,684 & 1,675 & $\ldots$ \\
\hline 1995 & 7,769 & 4,028 & $\ldots$ & 25,225 & 148 & $\ldots$ & $\ldots$ & 4,438 & 1,780 & $\ldots$ \\
\hline 1996 & 8,169 & 4,841 & 4,922 & 24,205 & 142 & $\ldots$ & $\ldots$ & 4,380 & 1,800 & $\ldots$ \\
\hline 1997 & 8,257 & 4,941 & 7,645 & 24,705 & $\ldots$ & 1,251 & 5,070 & 5,097 & 1,249 & $\ldots$ \\
\hline
\end{tabular}

Source: Data provided by Djibouti authorities. 
Table 13. Djibouti: Postal and Telecommunication Tariffs in Force at the Beginning of the Year, 1992-97

(In Djibouti francs)

\begin{tabular}{|c|c|c|c|c|c|c|}
\hline & 1992 & 1993 & 1994 & 1995 & 1996 & 1997 \\
\hline \multicolumn{7}{|l|}{ Postal service } \\
\hline \multicolumn{7}{|l|}{ Private letter, domestic } \\
\hline Up to $20 \mathrm{~g}$ & 30 & 30 & 30 & 30 & 30 & 30 \\
\hline From $500 \mathrm{~g}$ to $1 \mathrm{~kg}$ & 290 & 290 & 290 & 290 & 290 & 290 \\
\hline \multicolumn{7}{|l|}{ Private letter by air } \\
\hline $10 \mathrm{~g}$ to France & 120 & 120 & 120 & 120 & 120 & 75 \\
\hline Registered mail & 300 & 420 & 420 & 420 & 420 & 375 \\
\hline \multicolumn{7}{|l|}{ Postal packet } \\
\hline Air, $1 \mathrm{~kg}$ to France & 3,211 & 3,211 & 3,200 & 1,410 & 1,410 & 1,130 \\
\hline \multicolumn{7}{|l|}{ Postal box (annual rent) } \\
\hline Small size & 5,000 & 5,000 & 5,000 & 5,000 & 5,000 & 5,000 \\
\hline Big size & 7,500 & 7,500 & 7,500 & 7,500 & 7,500 & 7,500 \\
\hline Giant size & 10,000 & 10,000 & 10,000 & 10,000 & 10,000 & 10,000 \\
\hline \multicolumn{7}{|l|}{ Telegraphic service } \\
\hline France (per word) & 56 & 56 & 56 & 56 & 56 & 56 \\
\hline Europe (Sweden) (per word) & 111 & 105 & 105 & 105 & 105 & 105 \\
\hline \multicolumn{7}{|l|}{ Telex } \\
\hline Tariff per unit & 643 & 643 & 643 & 638 & 578 & $2,943 \quad 1 /$ \\
\hline \multicolumn{7}{|l|}{ Telephone } \\
\hline Main subscription & 25,000 & 25,000 & 25,000 & 25,000 & 25,000 & 25,000 \\
\hline Urban communication & 50 & 35 & 50 & 35 & 35 & 35 \\
\hline International communication $2 /$ & 35 & 35 & 35 & 35 & 35 & 35 \\
\hline
\end{tabular}

Source: Data provided by the Djibouti authorities.

1/ Per 3 minutes.

$2 /$ The unit is variable (from 2 to 10 seconds) according to the country called. 
Table 14. Djibouti: Tourism, 1992-95

\begin{tabular}{lcccc}
\hline & 1992 & 1993 & 1994 & $19951 /$ \\
\hline Number of rooms & 330 & 330 & 330 & 330 \\
Number of beds & 546 & 545 & 545 & 545 \\
& & & & \\
Days multiplied by rooms & 120.8 & 120.5 & 120.5 & 120.5 \\
Days multiplied by beds & 199.8 & 198.9 & 198.9 & 198.9 \\
Number of tourists & 27.6 & 24.7 & 46.6 & 20.0 \\
Number of nights sold & 79.4 & 98.1 & 69.1 & 81.3 \\
& & & & \\
\hline
\end{tabular}

Source: Data provided by the Djibouti authorities.

1/ Latest data available. 
Table 15. Djibouti: Expatriate Consumer Price Index by Product Group, 1992-97

$$
(1989=100)
$$

\begin{tabular}{lrrrrrrr}
\hline & $\begin{array}{r}\text { Percentage } \\
\text { Weight }\end{array}$ & 1992 & 1993 & 1994 & 1995 & 1996 & $\begin{array}{r}\text { Prel. } \\
\text { Est. }\end{array}$ \\
& & 117.6 & 122.8 & 130.8 & 137.2 & 142.9 & 145.8 \\
\hline General index & & 3.4 & 4.4 & 6.5 & 4.9 & 4.2 & 2.0 \\
Percentage change & 41.0 & 123.3 & 128.1 & 132.9 & 143.7 & 151.0 & 152.2 \\
Food & 6.0 & 102.2 & 102.2 & 113.3 & 113.3 & 113.3 & 113.4 \\
Water, gas, electricity & 6.0 & 127.2 & 127.2 & 127.2 & 127.2 & 127.2 & 127.4 \\
Domestic help & 14.0 & 116.8 & 126.8 & 141.1 & 147.0 & 151.5 & 151.9 \\
Maintenance & 17.0 & 109.8 & 114.9 & 133.6 & 134.8 & 139.2 & 139.7 \\
Transport and tourism & 16.0 & 113.2 & 119.2 & 120.6 & 126.5 & 134.8 & 135.2 \\
Miscellaneous & & & & & & & \\
\hline
\end{tabular}

Source: Data provided by the Djibouti authorities. 
Table 16. Djibouti: Demand for Employment and Registered Offers at the National Employment Service, 1992-97

\begin{tabular}{lrrrrrr}
\hline & 1992 & 1993 & 1994 & 1995 & 1996 & 1997 \\
& 3,992 & 4,299 & 5,176 & 4,927 & 3,780 & 2,405 \\
New demands & 899 & 447 & 1,340 & 1,414 & 836 & 604 \\
Offers & $-3,093$ & $-3,852$ & $-3,836$ & $-3,513$ & $-2,944$ & $-1,801$ \\
$\begin{array}{l}\text { Difference } \\
\begin{array}{l}\text { Sum of nonsatisfied } \\
\text { demands since 1977 }\end{array}\end{array}$ & 54,282 & 58,134 & 61,970 & 65,483 & 68,427 & 70,228 \\
\end{tabular}

Source: Data provided by the Djibouti authorities. 
Table 17. Djibouti: Number of Government Employees, 1992-97

\begin{tabular}{lrrrrrr}
\hline & 1992 & 1993 & 1994 & 1995 & 1996 & 1997 \\
\hline $\begin{array}{c}\text { Total number of } \\
\text { government employees 1/ } \\
\text { (percentage change) }\end{array}$ & 12,098 & 12,336 & 12,517 & 12,551 & 11,572 & 11,155 \\
Of which & 0.3 & 2.0 & 1.5 & 0.3 & -7.8 & -3.6 \\
Interior & & & & & & \\
$\quad$ Percent of total & 2,323 & 2,333 & 2,333 & 2,346 & 2,334 & 2,334 \\
National education & 19.2 & 18.9 & 18.6 & 18.7 & 20.2 & 20.9 \\
$\quad$ Percent of total & 1,448 & 1,534 & 1,550 & 1,618 & 1,739 & 1,756 \\
Health & 12.0 & 12.4 & 12.4 & 12.9 & 15.0 & 15.7 \\
Percent of total & 1,024 & 1,067 & 1,067 & 1,152 & 1,168 & 1,175 \\
Public works & 8.5 & 8.6 & 8.5 & 9.2 & 10.1 & 10.5 \\
$\quad$ Percent of total & 401 & 405 & 406 & 551 & 551 & 327 \\
Agriculture & 3.3 & 3.3 & 3.2 & 4.4 & 4.8 & 2.9 \\
Percent of total & 342 & 356 & 356 & 422 & 357 & 294 \\
\hline
\end{tabular}

Source: Data provided by the $\mathrm{D}_{\mathrm{j} i b o u t i}$ authorities; and Fund staff estimates.

1/ Including the army, the police, and contract personnel, but excluding mobilized personnel. 
Table 18. Djibouti: Employment and Wage Bill, 1992-97

(In millions of Djibouti francs, except as noted)

$\begin{array}{llllll}1992 & 1993 & 1994 & 1995 & 1996 & 1997\end{array}$

\section{Central Government}

Number of employees $1 /$

Percentage change

Wage bill 1/

Average annual wage

Private sector and parastatals

Number of employees

Wage bill

Average annual wage (thousands)
$12,098 \quad 12,336$

$0.3 \quad 2.0$

12,517

1.5

12,551

11,572

$-7.8 \quad-3.6$

15,350

15,766

16,026

17,330

14,502

15,423

1.3

1.3

1.3

1.4

1.3

1.4
17,612

12,208

727

Sources: Data provided by the Djibouti authorities; and Fund staff estimates.

1/ Including the army, the police, and contract personnel, but excluding mobilized personnel. 
Table 19. Djibouti: Public Investment Program, 1992-97

(In millions of Djibouti francs)

\begin{tabular}{|c|c|c|c|c|c|c|}
\hline & 1992 & 1993 & 1994 & 1995 & 1996 & $\begin{array}{l}\text { Prel. } \\
\text { Est. } \\
1997\end{array}$ \\
\hline Primary sector & 540 & 950 & 743 & 1,964 & 1,213 & 729 \\
\hline Agriculture & 200 & 225 & 108 & 209 & 93 & 50 \\
\hline Livestock and fishing & 49 & 52 & 78 & 751 & 683 & 680 \\
\hline Rural water and power & 291 & 673 & 557 & 1,004 & 437 & 0 \\
\hline Secondary sector & 2,191 & 1,055 & 1,569 & 1,936 & 953 & 1,040 \\
\hline Industry & $\ldots$ & $\ldots$ & $\ldots$ & $\ldots$ & $\ldots$ & -- \\
\hline Mining & 93 & 139 & - & - & -- & - \\
\hline Water & 2,013 & 811 & 921 & 464 & 344 & 309 \\
\hline Electricity & 77 & 28 & 482 & 1,387 & 609 & 731 \\
\hline Handicraft & 8 & 77 & 166 & 85 & -- & 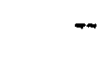 \\
\hline Infrastructure & 9,659 & 8,413 & 5,997 & 5,092 & 4,268 & 4,825 \\
\hline Roads & 3,253 & 2,640 & 1,907 & 590 & 111 & 415 \\
\hline Port & 4,921 & 2,995 & 2,497 & 2,498 & 1,699 & 1359 \\
\hline Air transport & .777 & 1,434 & 603 & 149 & 109 & -- \\
\hline Post and telecommunications & 35 & 270 & 673 & 260 & 336 & 302 \\
\hline Construction & 121 & 15 & 16 & 574 & -- & \\
\hline Urban development & 552 & 1,060 & 301 & 1,021 & 2,013 & 2748 \\
\hline Tourism & $\ldots$ & $\ldots$ & $\cdots$ & $\ldots$ & $\ldots$ & - \\
\hline Social sectors & $.3,140$ & 4,258 & 3,733 & 2,024 & 831 & 1,006 \\
\hline Population and employment & 108 & 642 & 696 & 42 & 85 & 120 \\
\hline Education and training & 1,471 & 1,832 & 2,316 & 1,302 & 345 & 464 \\
\hline Health & 431 & 532 & 277 & 356 & 301 & 315 \\
\hline Culture and sports & 532 & 699 & 69 & - & - & -- \\
\hline Social development and women & 295 & 496 & 336 & 228 & 100 & 107 \\
\hline Environment & -- & -- & -- & - & -- & -- \\
\hline Communication & 239 & 26 & 11 & 96 & -- & -- \\
\hline Other & 64 & 32 & 28 & - & -- & - \\
\hline \multicolumn{7}{|l|}{ Administration, planning, } \\
\hline and finance & 2,288 & 2,713 & 2,504 & 1,172 & 697 & 447 \\
\hline Economic management & 1,525 & 1,679 & 1,422 & 910 & 697 & 447 \\
\hline Administrative equipment & 763 & 1,034 & 1,082 & 262 & - & \\
\hline Total & 17,818 & 17,390 & 14,546 & 12,188 & 7,962 & 8047 \\
\hline Public enterprises & 10,021 & 10,524 & 9,742 & 8,982 & 4,531 & 4,211 \\
\hline
\end{tabular}

Sources: Data provided by the Djibouti authorities; and Fund staff estimates. 
Table 20. Djibouti: Consolidated Fiscal Operations, 1992-97

(In millions of Djibouti francs)

\begin{tabular}{|c|c|c|c|c|c|c|}
\hline & 1992 & 1993 & 1994 & 1995 & 1996 & $\frac{\text { Prel. }}{1997}$ \\
\hline Revenue & 22,463 & 23,751 & 25,591 & 25,390 & 25,395 & 24,408 \\
\hline Tax revenue & 21,193 & 22,461 & 24,320 & 23,569 & 23,486 & 21,881 \\
\hline Nontax revenue & 1,270 & 1,290 & 1,271 & 1,821 & 1,909 & 2,527 \\
\hline Total expenditure & 42,580 & 41,982 & 37,217 & 34,242 & 30,430 & 31,649 \\
\hline Current expenditure & 34,783 & 35,116 & 32,409 & 31,036 & 26,999 & 27,813 \\
\hline Wages and salaries & 19,838 & 20,135 & 20,997 & 21,107 & 18,348 & 19,701 \\
\hline Material and supplies & 9,636 & 9,523 & 5,740 & 6,441 & 4,268 & 4,400 \\
\hline Maintenance & 657 & 555 & 635 & 454 & 422 & 453 \\
\hline Other $1 /$ & 2,108 & 2,162 & 2,535 & 3,034 & 3,961 & 3,259 \\
\hline Capital expenditure & 7,797 & 6,866 & 4,808 & 3,206 & 3,431 & 3,836 \\
\hline Balance (payment-order) & $-20,117$ & $-18,231$ & $-11,626$ & $-8,852$ & $-5,035$ & $-7,241$ \\
\hline Arrears (decrease $=-$ ) & 287 & 3,131 & 1,984 & 2,772 & -603 & 953 \\
\hline Balance (cash-basis) & $-19,830$ & $-15,100$ & $-9,642$ & $-6,080$ & $-5,636$ & $-6,288$ \\
\hline Financing & 19,830 & 15,100 & 9,642 & 6,080 & 5,638 & 6,288 \\
\hline Domestic financing & 8,021 & 4,899 & 3,204 & 2,887 & 1,085 & 405 \\
\hline Bank financing & 5,690 & 1,043 & 1,060 & 1,121 & 51 & 880 \\
\hline Nonbank financing & 2,331 & 3,857 & 2,144 & $1,766^{\circ}$ & 1,034 & -475 \\
\hline External financing & 11,809 & 10,201 & 6,438 & 3,193 & 4,553 & 3,802 \\
\hline Grants & 10,366 & 7,382 & 4,314 & 1,779 & 1,611 & 2,294 \\
\hline Net borrowing & 43 & 2,554 & 2,063 & $-1,856$ & 2,835 & 1,492 \\
\hline Debt regularization & 0 & 0 & 0 & 3,270 & 107 & 16 \\
\hline External financing gap & 0 & 0 & 0 & 0 & 0 & 2,081 \\
\hline Financing secured & 0 & 0 & 0 & 0 & 0 & $2,0812 /$ \\
\hline Residual gap & 0 & 0 & 0 & 0 & 0 & 0 \\
\hline
\end{tabular}

Sources: Data provided by the Djibouti authorities; and Fund staff estimates.

1/ Includes estimates of extrabudgetary current outlays financed by external grants and loans.

2/ Comprising DF 786 million in grants for the demobilization program, DF 290 million in budgetary grants, and DF 1,005 million in debt regularization. 
Table 21. Djibouti: Government Revenue, 1992-97

(In millions of Djibouti francs)

\begin{tabular}{|c|c|c|c|c|c|c|}
\hline & 1992 & 1993 & 1994 & 1995 & 1996 & $\frac{\text { Prel. }}{1997}$ \\
\hline Total revenue & 22,463 & 23,751 & 25,591 & 25,390 & 25,395 & 24,408 \\
\hline Tax revenue & 21,193 & 22,461 & 24,320 & 23,569 & 23,486 & 21,881 \\
\hline Direct taxes & 8,728 & 9,186 & 9,843 & 9,434 & 10,409 & 9,560 \\
\hline Property tax & 723 & 911 & 794 & 828 & 1,078 & 932 \\
\hline Licenses & 1,345 & 1,405 & 1,207 & 1,159 & 1,454 & 1,312 \\
\hline Tax on wages and salaries & 3,067 & 2,719 & 3,162 & 3,098 & 3,675 & 3,262 \\
\hline Tax on income and profits & 1,588 & 1,673 & 2,024 & 1,741 & 1,627 & 1,426 \\
\hline Tax on companies & 1,299 & 1,151 & 1,581 & 1,318 & 1,271 & 1,062 \\
\hline Other (BIC, BNC, and PVI) & 288 & 522 & 444 & 423 & 356 & 364 \\
\hline Other & 224 & 286 & 615 & 711 & 339 & 564 \\
\hline Patriotic contribution & 1,782 & 2,193 & 2,042 & 1,897 & 2,236 & 2,064 \\
\hline Indirect taxes & 11,177 & 12,038 & 13,101 & 12,725 & 11,798 & 11,323 \\
\hline Domestic taxes & 8,049 & 8,042 & 8,154 & 7,858 & 7,438 & 7,117 \\
\hline Surcharge on Khat & 1,483 & 1,972 & 2,256 & 2,345 & 2,236 & 2,192 \\
\hline Surcharge on petroleum products & 455 & 413 & 1,073 & 1,091 & 1,041 & 1,021 \\
\hline Surcharge on tobacco & 513 & 707 & 682 & 592 & 467 & 251 \\
\hline Surcharge on alcohol & 413 & 603 & 650 & 537 & 500 & 484 \\
\hline Other surcharges & 266 & 301 & 285 & 302 & 116 & 258 \\
\hline Registration, tags and stamp duties & 1,287 & 1,238 & 1,376 & 1,411 & 1,279 & 998 \\
\hline Registration fees and tags & 573 & 584 & 582 & 788 & 595 & 527 \\
\hline Stamp duties & 560 & 506 & 409 & 302 & 388 & 471 \\
\hline Other & 154 & 147 & 386 & 321 & 296 & 0 \\
\hline Nontax revenue & 1,270 & 1,290 & 1,271 & 1,821 & 1,909 & 2,527 \\
\hline Estate revenue & 149 & 161 & 141 & 38 & 144 & 31 \\
\hline Service revenue & 398 & 483 & 356 & 266 & 283 & 289 \\
\hline Other & 551 & 439 & 489 & 1,021 & 929 & 1,623 \\
\hline Property sales & 172 & 207 & 285 & 496 & 69 & 0 \\
\hline Debt relief & $\ldots$ & $\ldots$ & $\ldots$ & $\ldots$ & 484 & 174 \\
\hline Other fees & $\ldots$ & $\ldots$ & $\ldots$ & $\ldots$ & $\ldots$ & 290 \\
\hline Reimbursement of family benefits & $\ldots$ & $\ldots$ & $\ldots$ & $\ldots$ & $\ldots$ & 120 \\
\hline
\end{tabular}

Sources: Data provided by the Djibouti authorities; and Fund staff estimates. 
Table 22. Djibouti: Government Revenue, 1992-97

(In percent of GDP)

\begin{tabular}{|c|c|c|c|c|c|c|}
\hline & 1992 & 1993 & 1994 & 1995 & 1996 & $\frac{\text { Prel. }}{1997}$ \\
\hline Total revenue & 26.9 & 28.4 & 29.6 & 29.1 & 29.5 & 27.5 \\
\hline Tax revenue & 25.4 & 26.8 & 28.1 & 27.0 & 27.2 & 24.6 \\
\hline Direct taxes & 10.5 & 11.0 & 11.4 & 10.8 & 12.1 & 10.8 \\
\hline Property tax & 0.9 & 1.1 & 0.9 & 0.9 & 1.3 & 1.0 \\
\hline Licenses & 1.6 & 1.7 & 1.4 & 1.3 & 1.7 & 1.5 \\
\hline Tax on wages and salaries & 3.7 & 3.2 & 3.7 & 3.6 & 4.3 & 3.7 \\
\hline Tax on income and profits & 1.9 & 2.0 & 2.3 & 2.0 & 1.9 & 1.6 \\
\hline Tax on companies & 1.6 & 1.4 & 1.8 & 1.5 & 1.5 & 1.2 \\
\hline Other (BIC, BNC, and PVI) & 0.3 & 0.6 & 0.5 & 0.5 & 0.4 & 0.4 \\
\hline Other & 0.3 & 0.3 & 0.7 & 0.8 & 0.4 & 0.6 \\
\hline Patriotic contribution & 2.1 & 2.6 & 2.4 & 2.2 & 2.6 & 2.3 \\
\hline Indirect taxes & 13.4 & 14.4 & 15.1 & 14.6 & 13.7 & 12.7 \\
\hline Domestic taxes & 9.6 & 9.6 & 9.4 & 9.0 & 8.6 & 8.0 \\
\hline Surcharge on Khat & 1.8 & 2.4 & 2.6 & 2.7 & 2.6 & 2.5 \\
\hline Surcharge on petroleum products & 0.5 & 0.5 & 1.2 & 1.3 & 1.2 & 1.1 \\
\hline Surcharge on tobacco & 0.6 & 0.8 & 0.8 & 0.7 & 0.5 & 0.3 \\
\hline Surcharge on alcohol & 0.5 & 0.7 & 0.8 & 0.6 & 0.6 & 0.5 \\
\hline Other surcharges & 0.3 & 0.4 & 0.3 & 0.3 & 0.1 & 0.3 \\
\hline Registration, tags and stamp duties & 1.5 & 1.5 & 1.6 & 1.6 & 1.7 & 1.1 \\
\hline Registration fees and tags & 0.7 & 0.7 & 0.7 & 0.9 & 0.7 & 0.6 \\
\hline Stamp duties & 0.7 & 0.6 & 0.5 & 0.3 & 0.5 & 0.5 \\
\hline Other & 0.2 & 0.2 & 0.4 & 0.4 & 0.3 & 0.0 \\
\hline Nontax revenue & 1.5 & 1.5 & 1.5 & 2.1 & 2.2 & 2.8 \\
\hline Estate revenue & 0.2 & 0.2 & 0.2 & - & 0.2 & 0.0 \\
\hline Service revenue & 0.5 & 0.6 & 0.4 & 0.3 & 0.3 & 0.3 \\
\hline Other & 0.7 & 0.5 & 0.6 & 1.2 & 1.1 & 1.8 \\
\hline Property sales & 0.2 & 0.2 & 0.3 & 0.6 & 0.1 & 0.0 \\
\hline Debt relief & $\ldots$ & $\ldots$ & $\ldots$ & $\ldots$ & $\ldots$ & 0.2 \\
\hline Other fees & $\ldots$ & $\ldots$ & $\ldots$ & $\ldots$ & $\ldots$ & 0.3 \\
\hline Reimbursement of family benefits & $\ldots$ & $\ldots$ & $\ldots$ & $\ldots$ & $\ldots$ & 0.1 \\
\hline
\end{tabular}

Sources: Data provided by the Djibouti authorities; and Fund staff estimates. 
Table 23. Djibouti: Government Revenue, 1992-97

(In percent of total revenue)

\begin{tabular}{|c|c|c|c|c|c|c|}
\hline & 1992 & 1993 & 1994 & 1995 & 1996 & $\frac{\text { Prel. }}{1997}$ \\
\hline Total revenue & 100.0 & 100.0 & 100.0 & 100.0 & 100.0 & 100.0 \\
\hline Tax revenue & 94.3 & 94.6 & 95.0 & 92.8 & 92.5 & 89.6 \\
\hline Direct taxes & 38.9 & 38.7 & 38.5 & 37.2 & 41.0 & 39.2 \\
\hline Property tax & 3.2 & 3.8 & 3.1 & 3.3 & 4.2 & 3.8 \\
\hline Licenses & 6.0 & 5.9 & 4.7 & 4.6 & 5.7 & 5.4 \\
\hline Tax on wages and salaries & 13.7 & 11.4 & 12.4 & 12.2 & 14.5 & 13.4 \\
\hline Tax on income and profits & 7.1 & 7.0 & 7.9 & 6.9 & 6.4 & 5.8 \\
\hline Tax on companies & 5.8 & 4.8 & 6.2 & 5.2 & 5.0 & 4.4 \\
\hline Other (BIC, BNC, and PVI) & 1.3 & 2.2 & 1.7 & 1.7 & 1.4 & 1.5 \\
\hline Other & 1.0 & 1.2 & 2.4 & 2.8 & 1.3 & 2.3 \\
\hline Patriotic contribution & 7.9 & 9.2 & 8.0 & 7.5 & 8.8 & 8.5 \\
\hline Indirect taxes & 49.8 & 50.7 & 51.2 & 50.1 & 46.5 & 46.4 \\
\hline Domestic taxes & 35.8 & 33.9 & 31.9 & 30.9 & 29.3 & 29.2 \\
\hline Surcharge on Khat & 6.6 & 8.3 & 8.8 & 9.2 & 8.8 & 9.0 \\
\hline Surcharge on petroleum products & 2.0 & 1.7 & 4.2 & 4.3 & 4.1 & 4.2 \\
\hline Surcharge on tobacco & 2.3 & 3.0 & 2.7 & 2.3 & 1.8 & 1.0 \\
\hline Surcharge on alcohol & 1.8 & 2.5 & 2.5 & 2.1 & 2.0 & 2.0 \\
\hline Other surcharges & 1.2 & 1.3 & 1.1 & 1.2 & 0.5 & 1.1 \\
\hline Registration, tags and stamp duties & 5.7 & 5.2 & 5.4 & 5.6 & 5.0 & 4.1 \\
\hline Registration fees and tags & 2.6 & 2.5 & 2.3 & 3.1 & 2.3 & 2.2 \\
\hline Stamp duties & 2.5 & 2.1 & 1.6 & 1.2 & 1.5 & 1.9 \\
\hline Other & 0.7 & 0.6 & 1.5 & 1.3 & $1: 2$ & 0.0 \\
\hline Nontax revenue & 5.7 & 5.4 & 5.0 & 7.2 & 7.5 & 10.4 \\
\hline Estate revenue & 0.7 & 0.7 & 0.6 & 0.1 & 0.6 & 0.1 \\
\hline Service revenue & 1.8 & 2.0 & 1.4 & 1.0 & 1.1 & 1.2 \\
\hline Other & 2.5 & 1.8 & 1.9 & 4.2 & 3.7 & 6.6 \\
\hline Property sales & 0.8 & 0.9 & 1.1 & 2.0 & 0.3 & 0.0 \\
\hline Debt relief & $\ldots$ & $\cdots$ & $\ldots$ & $\ldots$ & $\ldots$ & 0.7 \\
\hline Other fees & $\ldots$ & $\ldots$ & $\ldots$ & $\ldots$ & $\ldots$ & 1.2 \\
\hline Reimbursement of family benefits & $\cdots$ & $\ldots$ & $\ldots$ & $\ldots$ & $\ldots$ & 0.5 \\
\hline
\end{tabular}

Sources: Data provided by the Djibouti authorities; and Fund staff estimates. 
Table 24. Djibouti: Government Expenditure, 1992-97

(In millions of Djibouti francs)

\begin{tabular}{|c|c|c|c|c|c|c|}
\hline & 1992 & 1993 & 1994 & 1995 & 1996 & $\frac{\text { Prel. }}{1997}$ \\
\hline Total expenditure & 42,580 & 41,982 & 37,217 & 34,242 & 30,430 & 31,649 \\
\hline Current expenditure & 34,783 & 35,116 & 32,409 & 31,036 & 26,999 & 27,813 \\
\hline Treasury & 33,278 & 33,625 & 31,467 & 30,762 & 25,753 & 27,813 \\
\hline Wages and salaries & 19,838 & 20,135 & 20,997 & 21,108 & 18,348 & 19,701 \\
\hline Budget allocation & 15,578 & 15,925 & 16,026 & 16,607 & 14,991 & 16,658 \\
\hline General mobilization/demobilization & 4,260 & 4,210 & 4,971 & 4,501 & 3,357 & 3,043 \\
\hline Mobilization & $\ldots$ & $\ldots$ & 4,637 & 4,037 & 3,207 & 2,434 \\
\hline Demobilization & -- & -- & 334 & 464 & 150 & 609 \\
\hline Material and supplies & 9,636 & 9,523 & 5,740 & 6,441 & 4,268 & 4,400 \\
\hline Budget allocation & 7,232 & 6,358 & 5,159 & 6,067 & $\ldots$ & $\ldots$ \\
\hline General mobilization & 2,403 & 3,165 & 581 & 374 & $\ldots$ & \\
\hline Maintenance & 657 & 555 & 635 & 454 & 422 & 453 \\
\hline Transfers & 2,034 & 2,375 & 2,131 & 1,735 & 2,109 & 2,518 \\
\hline International organizations & 140 & 80 & 193 & 90 & 152 & 100 \\
\hline Special treasury accounts & 170 & 120 & 153 & 132 & 78 & 127 \\
\hline Publics entities & 276 & 563 & 543 & 323 & 312 & 692 \\
\hline Private entities & 251 & 149 & 138 & 147 & 107 & 155 \\
\hline Subsidies & 248 & 248 & 113 & 150 & 150 & 409 \\
\hline Scholarships & 755 & 1,071 & 817 & 859 & 830 & 642 \\
\hline Other & 36 & 71 & 42 & 35 & 480 & 393 \\
\hline Reduction in arrears & 159 & 73 & 134 & $\ldots$ & $\ldots$ & \\
\hline Other & 32 & 9 & 21 & & 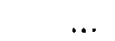 & 285 \\
\hline Interest on public debt & 510 & 366 & 371 & 602 & 606 & 456 \\
\hline External debt & 361 & 167 & 146 & 151 & 237 & 200 \\
\hline Domestic debt & 106 & 178 & 145 & 370 & 255 & 256 \\
\hline Certificates of deposits & 43 & 21 & 80 & 80 & 114 & $\ldots$ \\
\hline Other-Adjustment & 572 & 662 & 1,572 & 875 & 581 & 0 \\
\hline Extra-treasury & 1,505 & 1,491 & 942 & 274 & 665 & 0 \\
\hline Capital expenditure $1 /$ & 7,796 & 6,865 & 4,808 & 3,206 & 3,431 & 3,836 \\
\hline Domestically financed & 1,777 & 903 & 1,038 & 735 & 444 & 584 \\
\hline Budgetary & 1,343 & 502 & 835 & $\ldots$ & $\ldots$ & ... \\
\hline Food aid proceeds & 102 & 104 & 18 & $\ldots$ & $\ldots$ & $\ldots$ \\
\hline Special accounts & 332 & 298 & 185 & $\ldots$ & 93 & \\
\hline Foreign financed & 6,019 & 5,962 & 3,770 & 2,470 & 2,894 & 3,252 \\
\hline \multicolumn{7}{|l|}{ Functional classification } \\
\hline Current expenditure & 33,278 & 33,625 & 31,467 & 31,036 & 26,999 & 27,813 \\
\hline General administration & 12,263 & 12,073 & 10,905 & 12,304 & 10,271 & 11,122 \\
\hline Defense & 5,089 & 4,702 & 4,648 & 4,481 & 3,712 & 4,019 \\
\hline Mobilization/demobilization & 6,663 & 7,375 & 5,553 & 4,875 & 3,357 & 3,635 \\
\hline Social services & 4,429 & 4,488 & 4,509 & 4,855 & 4,486 & 4,858 \\
\hline Education & 2,379 & 2,575 & 2,615 & 3,202 & 2,990 & 3,238 \\
\hline Health & 2,050 & 1,913 & 1,894 & 1,653 & 1,496 & 1,620 \\
\hline Economic services & 1,687 & 1,575 & 1,758 & 1,033 & 850 & 920 \\
\hline Agriculture & 461 & 483 & 535 & 552 & 450 & 487 \\
\hline Public works & 569 & 538 & 588 & 481 & 400 & 433 \\
\hline Maintenance work & 657 & 555 & 635 & & & \\
\hline Transfers & 2,003 & 2,366 & 2,110 & 1,735 & 2,109 & 2,518 \\
\hline Other & 32 & 9 & 21 & & & 285 \\
\hline Interest on public debt & 510 & 366 & 371 & 602 & 606 & 456 \\
\hline Other-Adjustment & 572 & 662 & 1,572 & 1,151 & 1,608 & 0 \\
\hline
\end{tabular}

Sources: Data provided by the Djibouti authorities; and Fund staff estimates.

1/ Prior to 1997, the treasury did not include foreign-financed capital expenditure in its accounts; consequently the distinction was between treasury and extra-treasury capital expenditure. 
Table 25. Djibouti: Government Expenditure, 1992-97

(In percent of GDP)

\begin{tabular}{|c|c|c|c|c|c|c|}
\hline & 1992 & 1993 & 1994 & 1995 & 1996 & $\frac{\text { Prel. }}{1997}$ \\
\hline Total expenditure & 51.0 & 50.1 & 43.0 & 39.2 & 35.3 & 35.6 \\
\hline Current expenditure & 41.7 & 41.9 & 37.4 & 35.6 & 31.3 & 31.3 \\
\hline Treasury & 39.9 & 40.1 & 36.3 & 35.3 & 30.5 & 31.3 \\
\hline Wages and salaries & 23.8 & 24.0 & 24.2 & 24.2 & 21.3 & 22.2 \\
\hline Budget allocation & 18.7 & 19.0 & 18.5 & & 17.4 & 18.7 \\
\hline General mobilization/demobilization & 5.1 & 5.0 & 5.7 & 5.2 & 3.9 & 2.7 \\
\hline Mobilization & $\ldots$ & $\ldots$ & 5.4 & 4.6 & 3.7 & 0.7 \\
\hline Demobilization & $\because$ & -- & 0.4 & 0.6 & 0.2 & 0.7 \\
\hline Material and supplies & 11.5 & 11.4 & 6.6 & 7.4 & 5.0 & 4.9 \\
\hline Budget allocation & 8.7 & 7.6 & 6.0 & 7.0 & $\ldots$ & \\
\hline General mobilization & 2.9 & 3.8 & 0.7 & 0.4 & $\cdots$ & \\
\hline Maintenance & 0.8 & 0.7 & 0.7 & 0.5 & 0.5 & 0.5 \\
\hline Transfers & 2.4 & 2.8 & 2.5 & 2.0 & 2.4 & 2.8 \\
\hline International organizations & 0.2 & 0.1 & 0.2 & 0.1 & 0.2 & 0.1 \\
\hline Special treasury accounts & 0.2 & 0.1 & 0.2 & 0.2 & 0.1 & 0.1 \\
\hline Publics entities & 0.3 & 0.7 & 0.6 & 0.4 & 0.4 & 0.8 \\
\hline Private entities & 0.3 & 0.2 & 0.2 & 0.2 & 0.1 & 0.2 \\
\hline Subsidies & 0.3 & 0.3 & 0.1 & 0.2 & 0.2 & 0.5 \\
\hline Scholarships & 0.9 & 1.3 & 0.9 & 1.0 & 1.0 & 0.7 \\
\hline Other & -. & 0.1 & - & - & 0.5 & 0.4 \\
\hline Reduction in arrears & 0.2 & 0.1 & 0.2 & -. & -. & \\
\hline Other & -- & - & - & -- & -- & 0.3 \\
\hline Interest on public debt & 0.6 & 0.4 & 0.4 & 0.7 & 0.7 & 0.5 \\
\hline External debt & 0.4 & 0.2 & 0.2 & 0.2 & 0.3 & 0.2 \\
\hline Domestic debt & 0.1 & 0.2 & 0.2 & 0.4 & 0.3 & 0.3 \\
\hline Certificates of deposits & 0.1 &.- & 0.1 & 0.1 & 0.1 & \\
\hline Other-Adjustment & 0.7 & 0.8 & 1.8 & 0.3 & 0.7 & 0.0 \\
\hline Extra-treasury & 1.8 & 1.8 & 1.1 & 0.3 & 0.8 & 0.0 \\
\hline Capital expenditure & 9.3 & 8.2 & 5.6 & 3.7 & 4.0 & 4.3 \\
\hline Treasury & 2.1 & 1.1 & 1.2 & 0.8 & 0.6 & 0.7 \\
\hline Budgetary & 2.1 & 1.1 & 1.2 & 0.8 & 0.6 & $\ldots$ \\
\hline Food aid proceeds & 0.1 & 0.1 & -- & .0 & & $\ldots$ \\
\hline Special accounts & 0.4 & 0.4 & 0.2 & $\ldots$ & 0.1 & \\
\hline Extra-treasury & 7.2 & 7.1 & 4.4 & 2.8 & 3.4 & 3.7 \\
\hline \multicolumn{7}{|l|}{ Functional classification } \\
\hline Current expenditure (treasury) & 41.7 & 41.9 & 37.4 & 35.6 & 31.3 & 31.3 \\
\hline General administration & 14.7 & 14.4 & 12.6 & $\ldots$ & 11.9 & 12.5 \\
\hline Defense & 6.1 & 5.6 & 5.4 & & 4.3 & 4.5 \\
\hline Mobilization/demobilization & 8.0 & 8.8 & 6.4 & 5.6 & 4.1 & 4.1 \\
\hline Social services & 5.3 & 5.4 & 5.2 & $\ldots$ & 5.2 & 5.5 \\
\hline Education & 2.8 & 3.1 & 3.0 & $\ldots$ & 3.5 & 3.6 \\
\hline Health & 2.5 & 2.3 & 2.2 & $\ldots$ & 1.7 & 1.8 \\
\hline Economic services & 2.0 & 1.9 & 2.0 & $\ldots$ & 1.0 & 1.0 \\
\hline Agriculture & 0.6 & 0.6 & 0.6 & $\ldots$ & 0.5 & 0.5 \\
\hline Public works & 0.7 & 0.6 & 0.7 & $\ldots$ & 0.5 & 0.5 \\
\hline Maintenance work & 0.8 & 0.7 & 0.7 & $\ldots$ & -. & \\
\hline Transfers & 2.4 & 2.8 & 2.4 & 2.0 & 2.4 & 2.8 \\
\hline Other & -- & -- & -- & & & 0.3 \\
\hline Interest on public debt & 0.6 & 0.4 & 0.4 & 0.7 & 0.7 & 0.5 \\
\hline Other-Adjustment & 0.7 & 0.8 & 1.8 & 1.3 & 1.7 & 0.0 \\
\hline
\end{tabular}

Sources: Data provided by the Djibouti authorities; and Fund staff estimates. 
Table 26. Djibouti: Government Expenditure, 1992-97

(In percent of total expenditure)

\begin{tabular}{|c|c|c|c|c|c|c|}
\hline & 1992 & 1993 & 1994 & 1995 & 1996 & $\frac{\text { Prel. }}{1997}$ \\
\hline Total expenditure & 100.0 & 100.0 & 100.0 & 100.0 & 100.0 & 100.0 \\
\hline Current expenditure & 81.7 & 83.6 & 87.1 & 90.6 & 88.7 & 87.9 \\
\hline Treasury & 78.2 & 80.1 & 84.5 & 89.8 & 86.5 & 87.9 \\
\hline Wages and salaries & 46.6 & 48.0 & 56.4 & 61.6 & 60.3 & 62.2 \\
\hline Budget allocation & 36.6 & 37.9 & 43.1 & 48.5 & 49.3 & 52.6 \\
\hline General mobilization/demobilization & 10.0 & 10.0 & 13.4 & 13.3 & 11.0 & 7.7 \\
\hline Mobilization & $\ldots$ & $\ldots$ & 11.7 & 11.7 & 10.5 & 1.9 \\
\hline Demobilization & -- & -- & 0.8 & 0.6 & 0.5 & 1.9 \\
\hline Material and supplies & 22.6 & 22.7 & 15.4 & 18.8 & 14.0 & 13.9 \\
\hline Budget allocation & 17.0 & 15.1 & 13.7 & 17.9 & $\ldots$ & $\ldots$ \\
\hline General mobilization & 5.6 & 7.5 & 1.5 & 0.9 & & \\
\hline Maintenance & 1.5 & 1.3 & 1.7 & 1.3 & 1.4 & 1.4 \\
\hline Transfers & 4.8 & 5.7 & 5.7 & 5.1 & 6.9 & 8.0 \\
\hline International organizations & 0.3 & 0.2 & 0.5 & 0.3 & 0.5 & 0.3 \\
\hline Special treasury accounts & 0.4 & 0.3 & 0.4 & 0.4 & 0.3 & 0.4 \\
\hline Publics entities & 0.6 & 1.3 & 1.4 & 0.9 & 1.0 & 2.2 \\
\hline Private entities & 0.6 & 0.4 & 0.4 & 0.4 & 0.4 & 0.5 \\
\hline Subsidies & 0.6 & 0.6 & 0.3 & 0.4 & 0.5 & 1.3 \\
\hline Scholarships & 1.8 & 2.6 & 2.2 & 2.5 & 2.7 & 2.0 \\
\hline Other & -- & 0.1 & 0.1 & 0.1 & 1.6 & 1.2 \\
\hline Reduction in arrears & 0.4 & 0.2 & 0.4 & $\ldots$ & $\ldots$ & \\
\hline Other & 0.1 & - & 0.1 & . & & 0.9 \\
\hline Interest on public debt & 1.2 & 0.9 & 1.0 & 1.8 & 2.0 & 1.4 \\
\hline External debt & 0.8 & 0.4 & 0.4 & 0.4 & 0.8 & 0.6 \\
\hline Domestic debt & 0.2 & 0.4 & 0.4 & 1.1 & 0.8 & 0.8 \\
\hline Certificates of deposits & 0.1 & 0.1 & 0.2 & 0.3 & 0.4 & \\
\hline Other-Adjustment & 1.4 & 1.7 & 5.2 & 2.6 & 1.9 & 0.0 \\
\hline Extra-treasury & 3.5 & 3.6 & 2.5 & 0.8 & 2.2 & 0.0 \\
\hline Capital expenditure & 18.3 & 16.3 & 12.8 & 9.4 & 11.3 & 12.1 \\
\hline Treasury & 4,2 & 2.2 & 2.8 & 2.1 & 1.8 & 1.8 \\
\hline Budgetary & 3.2 & 1.2 & 2.2 & ... & 1.5 & $\ldots$ \\
\hline Food grant proceeds & 0.2 & 0.2 & -- & $\ldots$ & & $\ldots$ \\
\hline Special accounts & 0.8 & 0.7 & 0.5 & $\ldots$ & 0.3 & \\
\hline Extra-treasury & 14.1 & 14.2 & 10.0 & 7.2 & 9.5 & 10.3 \\
\hline \multicolumn{7}{|l|}{ Functional classification } \\
\hline Current expenditure (treasury) & 81.7 & 83.6 & 87.1 & 90.6 & 88.7 & 87.9 \\
\hline General administration & 28.8 & 28.7 & 29.0 & $\ldots$ & 33.8 & 35.1 \\
\hline Defense & 12.0 & 11.2 & 12.4 & . & 12.2 & 12.7 \\
\hline Mobilization/demobilization & 15.6 & 17.6 & 14.8 & 14.3 & 11.6 & 11.5 \\
\hline Social services & 10.4 & 10.7 & 12.0 & $\ldots$ & 14.7 & 15.3 \\
\hline Education & 5.6 & 6.1 & 7.0 & $\ldots$ & 9.8 & 10.2 \\
\hline Health & 4.8 & 4.6 & 5.0 & $\ldots$ & 4.9 & 5.1 \\
\hline Economic services & 4.0 & 3.7 & 4.7 & $\ldots$ & 2.8 & 2.9 \\
\hline Agriculture & 1.1 & 1.1 & 1.4 & $\ldots$ & 1.5 & 1.5 \\
\hline Public works & 1.3 & 1.3 & 1.6 & $\ldots$ & 1.3 & 1.4 \\
\hline Maintenance work & 1.5 & 1.3 & 1.7 & 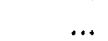 & & \\
\hline Transfers & 4.7 & 5.6 & 5.6 & 5.1 & 6.9 & 8.0 \\
\hline Other & 0.1 & -- & 0.1 & & & 0.9 \\
\hline Interest on public debt & 1.2 & 0.9 & 1.0 & 1.8 & 2.0 & 1.4 \\
\hline Other-Adjustment & 1.3 & 1.6 & 4.2 & 3.4 & 5.3 & 0.0 \\
\hline
\end{tabular}

Sources: Data provided by the Djibouti authorities; and Fund staff estimates. 
Table 27. Djibouti: Financing of Consolidated Fiscal Operations, 1992-97

(In millions of Djibouti francs)

\begin{tabular}{|c|c|c|c|c|c|c|}
\hline & 1992 & 1993 & 1994 & 1995 & 1996 & $\frac{\text { Prel }}{1997}$ \\
\hline \multicolumn{7}{|l|}{ Balance (payment-order basis and } \\
\hline excluding grants) & $-20,117$ & $-18,231$ & $-11,626$ & $-8,852$ & $-5,035$ & $-7,241$ \\
\hline Arrears (decrease $=-$ ) & 286 & 3,131 & 1,984 & 2,773 & -603 & 953 \\
\hline Domestic arrears & 286 & 3,092 & 993 & $\ldots$ & -717 & 1,987 \\
\hline Wages and salaries & - & 1,678 & -165 & $\ldots$ & -277 & 2,242 \\
\hline Goods and services & 286 & 1,414 & 1,158 & $\ldots$ & -440 & -255 \\
\hline On budget & 74 & 621 & 1,054 & $\ldots$ & -568 & -135 \\
\hline On special accounts & 213 & 806 & 104 & $\cdots$ & -128 & -120 \\
\hline External arrears & -. & 39 & 991 & $\ldots$ & 114 & $-1,034$ \\
\hline Balance (cash basis and excluding grants & $-19,830$ & $-15,100$ & $-9,642$ & $-6,079$ & $-5,638$ & $-6,288$ \\
\hline Financing & 19,830 & 15,100 & 9,642 & 6,079 & 5,638 & 6,288 \\
\hline Domestic financing & 8,021 & 4,899 & 3,204 & 2,887 & 1,085 & 405 \\
\hline Bank financing & 5,690 & 1,043 & 1,060 & 1,121 & 51 & 880 \\
\hline Central bank & 5,524 & 593 & 145 & 140 & 401 & 1,105 \\
\hline Claims on government & -. & 307 & 227 & - & 741 & 431 \\
\hline Government deposits & 5,524 & 286 & 82 & 140 & -340 & -674 \\
\hline Reserve fund & - & - & 132 & 135 & 0 & 0 \\
\hline Treasury cash in vaults & -853 & .81 & -3 & 5 & -8 & -6 \\
\hline Other & $-4,671$ & -205 & -47 & -- & -332 & -668 \\
\hline Commercial banks & 166 & 450 & 915 & 981 & -416 & -97 \\
\hline Claims on government & - & 446 & 2,079 & -381 & .257 & -134 \\
\hline Government deposits & -166 & 4 & $-1,164$ & 1,362 & -159 & 37 \\
\hline BDD & $\ldots$ & $\ldots$ & $\ldots$ & $\ldots$ & 66 & -128 \\
\hline Nonbank financing & 2,331 & 3,857 & 2,144 & 1,766 & 1,034 & -475 \\
\hline Public enterprises & 2,368 & 4,573 & 1,825 & 2,502 & 1,843 & - \\
\hline Short term deposits & 358 & 1,975 & 355 & 2,002 & 1,343 & - \\
\hline General mobilization & 700 & - & -. & - & - & - \\
\hline Budget contribution & - & 980 & 750 & 500 & 500 & -- \\
\hline Loans & 1,025 & 1,150 & 720 & - & - & - \\
\hline Advances & - & 400 & - & - & - & -- \\
\hline Certificates of deposits & 285 & 68 & -- & - & - & - \\
\hline Other public entities & 46 & 261 & -51 & 23 & -37 & - \\
\hline Customs duty bills & 74 & -475 & 654 & 13 & $\ldots$ & - \\
\hline Other & - & -- & 56 & 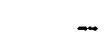 & -342 & $\ldots$ \\
\hline Amortization & 157 & 503 & 340 & 772 & 430 & -475 \\
\hline External financing & 11,809 & 10,201 & 6,438 & 3,193 & 4,553 & 5,883 \\
\hline Grants & 10,366 & 7,382 & 4,314 & 1,779 & 1,611 & 2,294 \\
\hline Treasury & 4,574 & 3,117 & 2,123 & 818 & 1,217 & 147 \\
\hline Extra-treasury & 5,792 & 4,265 & 2,191 & 961 & 394 & 2,147 \\
\hline Net borrowing & .43 & 2,554 & 2,063 & $-1,856$ & 2,835 & 1,492 \\
\hline Drawings & 1,732 & 3,188 & 2,521 & 1,784 & 3,125 & 1,671 \\
\hline Amortization & 1,689 & 634 & 458 & 3,640 & 290 & .179 \\
\hline Debt relief & 1,401 & 265 & 61 & 3,270 & 107 & 16 \\
\hline External financing gap & 0 & 0 & 0 & 0 & 0 & 2,081 \\
\hline Financing secured & 0 & 0 & 0 & 0 & 0 & 2,081 \\
\hline Residual gap & 0 & 0 & 0 & 0 & 0 & 0 \\
\hline \multicolumn{7}{|l|}{ Memorandum items } \\
\hline \multicolumn{7}{|l|}{ Balance (payment-order basis and } \\
\hline including grants) & $-9,751$ & $-10,849$ & $-7,312$ & $-7,033$ & $-3,424$ & $-4,947$ \\
\hline \multicolumn{7}{|l|}{ Overall balance (cash basis and } \\
\hline including grants) & $-9,465$ & $-7,718$ & $-5,328$ & $-4,300$ & $-4,027$ & $-3,994$ \\
\hline
\end{tabular}

Sources: Data provided by the Djibouti authorities; and Fund staff estimates. 
Table 28. Djibouti: Financing of Consolidated Fiscal Operations, 1992-97

(In percent of total financing)

\begin{tabular}{|c|c|c|c|c|c|c|}
\hline & 1992 & 1993 & 1994 & 1995 & 1996 & $\frac{\text { Prel: }}{1997}$ \\
\hline Overall financing & 100.0 & 100.0 & 100.0 & 100.0 & 100.0 & 100.0 \\
\hline Domestic financing & 40.4 & 32.4 & 33.2 & 47.5 & 19.2 & 6.4 \\
\hline Bank financing & 28.7 & 6.9 & 11.0 & 18.4 & 0.9 & 14.0 \\
\hline Central bank & 27.9 & 3.9 & 1.5 & 2.3 & 7.1 & 17.6 \\
\hline Claims on government & - & 2.0 & 2.4 & 0.0 & 13.1 & 6.9 \\
\hline Government deposits & -27.9 & -1.9 & 0.9 & -2.3 & 6.0 & -10.7 \\
\hline Reserve fund & -- & -- & 1.4 & -2.2 & 0.0 & 0.0 \\
\hline Treasury cash in vaults & -4.3 & -0.5 & -- & -0.1 & 0.0 & -0.1 \\
\hline Other & -23.6 & -1.4 & -0.5 & 0.0 & 0.0 & -10.6 \\
\hline Commercial banks & 0.8 & 3.0 & 9.5 & 16.1 & -7.4 & -1.5 \\
\hline Claims on government & - & 3.0 & 21.6 & -6.3 & 0.0 & -2.1 \\
\hline Government deposits & -0.8 & -. & 12.1 & -22.4 & -7.4 & 0.6 \\
\hline $\mathrm{BDD}$ & $\ldots$ & $\ldots$ & $\ldots$ & 0.0 & 1.2 & -2.0 \\
\hline Nonbank financing & 11.8 & 25.5 & 22.2 & 29.1 & 18.3 & -7.6 \\
\hline Public enterprises & 11.9 & 30.3 & 18.9 & 41.2 & 32.7 & -- \\
\hline Short term deposits & 1.8 & 13.1 & 3.7 & 32.9 & 23.8 & -- \\
\hline General mobilization & 3.5 & - & -. & 0.0 & 0.0 & -- \\
\hline Budget contribution & -- & 6.5 & 7.8 & 8.2 & 8.9 & -- \\
\hline Loans & 5.2 & 7.6 & 7.5 & 0.0 & 0.0 & - \\
\hline Advances & - & 2.6 & -- & 0.0 & 0.0 & -- \\
\hline Certificates of deposits & 1.4 & 0.5 & - & 0.0 & 0.0 & -- \\
\hline Other public entities & 0.2 & 1.7 & -0.5 & 0.4 & -0.8 & - \\
\hline Customs duty bills & 0.4 & -3.1 & 6.8 & 0.2 & 0.0 & -- \\
\hline Other & - & - & 0.6 & 0.0 & -6.1 & -- \\
\hline Amortization & 0.8 & 3.3 & 3.5 & 12.7 & 7.6 & -7.6 \\
\hline External financing & 59.6 & 67.6 & 66.8 & 52.5 & 80.8 & 93.6 \\
\hline Grants & 52.3 & 48.9 & 44.7 & 29.3 & 28.6 & 36.5 \\
\hline Treasury & 23.1 & 20.6 & 22.0 & 13.5 & 21.6 & 2.3 \\
\hline Extra-treasury & 29.2 & 28.2 & 22.7 & 15.8 & 7.0 & 34.1 \\
\hline Net borrowing & 0.2 & 16.9 & 21.4 & -30.5 & 50.3 & 23.7 \\
\hline Drawings & 8.7 & 21.1 & 26.1 & 29.3 & 55.4 & 26.6 \\
\hline Amortization & 8.5 & 4.2 & 4.7 & 59.9 & 5.1 & -2.8 \\
\hline Debt relief & 7.1 & 1.8 & 0.6 & 53.8 & 1.9 & 0.3 \\
\hline External financing gap & $\ldots$ & $\ldots$ & $\ldots$ & $\ldots$ & $\ldots$ & 33.1 \\
\hline Financing secured & $\ldots$ & $\ldots$ & $\ldots$ & $\ldots$ & $\ldots$ & 33.1 \\
\hline Residual gap & $\ldots$ & $\ldots$ & $\ldots$ & $\ldots$ & $\ldots$ & 0.0 \\
\hline
\end{tabular}

Sources: Data provided by the Djibouti authorities; and Fund staff estimates. 
Table 29. Djibouti: Financing of Consolidated Fiscal Operations, 1992-97

(In percent of GDP)

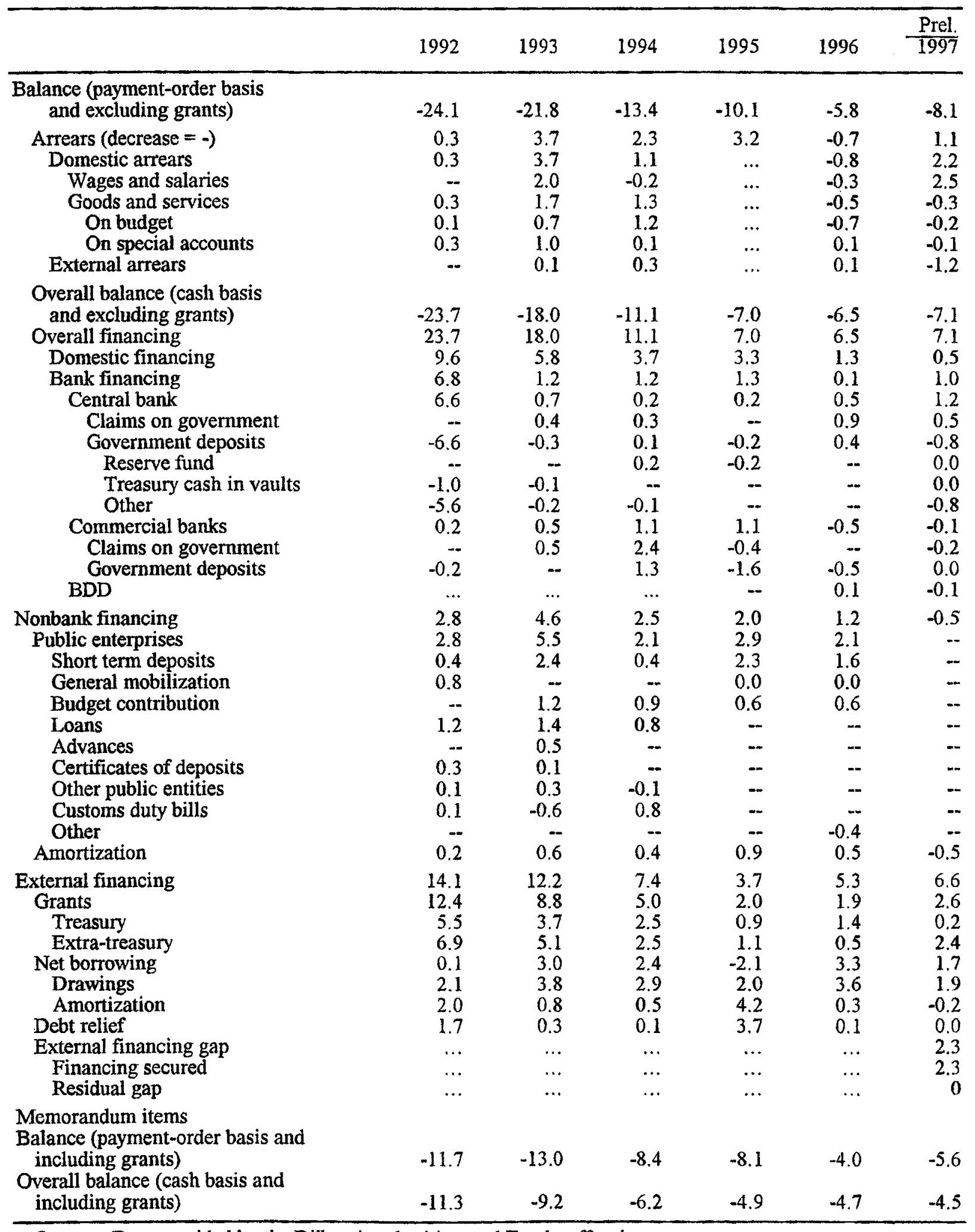

Sources: Data provided by the Djibouti authorities; and Fund staff estimates. 
Table 30. Djibouti: Factors Affecting Changes in Domestic Liquidity, 1992-97

\begin{tabular}{|c|c|c|c|c|c|c|}
\hline & 1992 & 1993 & 1994 & 1995 & 1996 & 1997 \\
\hline & \multicolumn{6}{|c|}{ (In millions of Djibouti francs; changes, December to December) } \\
\hline Net foreign assets & $-9,649$ & 1,863 & $-1,435$ & $-1,992$ & $-5,887$ & $-2,090$ \\
\hline Monetary authorities & $-2,966$ & $-1,478$ & 620 & $-1,277$ & 255 & $-2,017$ \\
\hline Commercial banks & $-6,683$ & 3,341 & $-2,055$ & -715 & $-6,142$ & -73 \\
\hline Net domestic assets & 5984 & $-3,070$ & 2,268 & 3,820 & 131 & 1,380 \\
\hline Domestic credit & 5178 & $-1,147$ & 1,725 & 5,589 & 1,147 & 886 \\
\hline \multicolumn{7}{|l|}{ Net credit to central } \\
\hline government & 5690 & 994 & 1,118 & 1,121 & -15 & 1,064 \\
\hline \multicolumn{7}{|l|}{ Credit to the rest of } \\
\hline the economy & -512 & $-2,141$ & 607 & 4,468 & 1,162 & -178 \\
\hline Other items (net) & 806 & $-1,923$ & 543 & $-1,769$ & $-1,016$ & 494 \\
\hline Money and quasi-money & -3665 & $-1,211$ & 835 & 1,828 & $-5,756$ & -710 \\
\hline Currency in circulation & 2,068 & -930 & 292 & $-1,326$ & 319 & -236 \\
\hline Demand deposits & 835 & 612 & -394 & -657 & $-2,419$ & $-1,232$ \\
\hline \multirow[t]{2}{*}{ Time deposits } & $-6,568$ & -893 & 937 & 3,811 & $-3,656$ & 758 \\
\hline & \multicolumn{6}{|c|}{$\begin{array}{l}\text { (Change from year earlier, as percent of broad } \\
\text { broad money at beginning of period) }\end{array}$} \\
\hline Net foreign assets & -16.2 & 3.3 & -2.6 & -3.6 & -10.3 & -4.0 \\
\hline Monetary authorities & -5.0 & -2.6 & 1.1 & -2.3 & 0.4 & -3.9 \\
\hline Commercial banks & -11.2 & 6.0 & -3.8 & -1.3 & -10.7 & -0.1 \\
\hline Net domestic assets & 10.0 & -5.5 & 4.1 & 6.9 & 0.2 & 2.7 \\
\hline Domestic credit & 8.7 & -2.7 & 3.1 & 10.1 & 2.0 & 1.7 \\
\hline \multicolumn{7}{|l|}{ Net credit to the central } \\
\hline government & 9.5 & 1.8 & 1.9 & 2.0 & - & 2.1 \\
\hline \multicolumn{7}{|l|}{ Credit to the rest of } \\
\hline the economy & -0.8 & -3.8 & 1.1 & 8.0 & 2.0 & -0.3 \\
\hline Other items net & -1.4 & 3.4 & -1.0 & -3.2 & -7.8 & 1.0 \\
\hline Money and quasi-money & -6.2 & -2.2 & 1.5 & 3.3 & -10.0 & -1.4 \\
\hline
\end{tabular}

Sources: Data provided by the Djibouti authorities; and Fund staff estimates. 
Table 31. Djibouti: Monetary Survey and Risk Indicators, 1992-97

\begin{tabular}{|c|c|c|c|c|c|c|}
\hline & 1992 & 1993 & 1994 & 1995 & 1996 & 1997 \\
\hline & \multicolumn{6}{|c|}{ (In millions of Djibouti francs) } \\
\hline Net foreign assets & 35,358 & 37,222 & 35,787 & 33,795 & 27,908 & 25,818 \\
\hline Monetary authorities & 14,806 & 13,328 & 13,948 & 12,671 & 12,926 & 10,909 \\
\hline Commercial banks & 20,552 & 23,894 & 21,839 & 21,124 & 14,982 & 14,909 \\
\hline Net domestic assets & 20,552 & 17,482 & 19,750 & 23,570 & 23,701 & 25,081 \\
\hline Domestic credit & 33,544 & 32,398 & 34,122 & 39,711 & 40,858 & 41,744 \\
\hline \multicolumn{7}{|l|}{ Net credit to the central } \\
\hline government & $-1,885$ & -891 & 227 & 1,348 & 1,333 & 2,397 \\
\hline Credit to the private sector & 35,429 & 33,288 & 33,895 & 38,363 & 39,525 & 39,347 \\
\hline Other items (net) & $-12,991$ & $-14,915$ & $-14,372$ & $-16,141$ & 17,157 & 16,663 \\
\hline Money and quasi-money & 55,912 & 54,704 & 55,537 & 57,365 & 51,609 & 50,899 \\
\hline Currency in circulation & 11,331 & 10,401 & 10,693 & 9,367 & 9,686 & 9,450 \\
\hline Demand deposits & 21,594 & 22,209 & 21,814 & 21,157 & 18,738 & 17,506 \\
\hline \multirow[t]{2}{*}{ Time deposits } & 22,986 & 22,094 & 23,530 & 26,841 & 23,185 & 23,943 \\
\hline & \multicolumn{6}{|c|}{ (In percent) } \\
\hline \multicolumn{7}{|l|}{ Risk indicators } \\
\hline \multicolumn{7}{|l|}{ Share of non-performing loans } \\
\hline in total loans & 13.2 & 12.7 & 13.1 & 13.9 & 14.6 & 14.9 \\
\hline \multicolumn{7}{|l|}{ Share of foreign exchange loans } \\
\hline in total lending & 21.1 & 15.9 & 16.8 & 24.1 & 23.8 & 20.4 \\
\hline \multicolumn{7}{|l|}{ Share of foreign exchange } \\
\hline deposits in total deposits & 37.4 & 44.7 & 44.0 & 51.9 & 48.8 & 56.2 \\
\hline \multicolumn{7}{|l|}{ Share of foreign currency-denominated } \\
\hline liabilities in total liabilities & 36.9 & 41.0 & 39.3 & 46.7 & 46.6 & 50.3 \\
\hline \multicolumn{7}{|l|}{ Share of foreign currency-denominated } \\
\hline assets in total assets & 60.0 & 60.1 & 59.0 & 60.0 & 56.6 & 54.2 \\
\hline Share of housing loans in total loans & $\ldots$ & 7.9 & 12.6 & 11.4 & 8.1 & 8.9 \\
\hline
\end{tabular}

Sources: Data provided by the Djibouti authorities; and Fund staff estimates. 
Table 32. Djibouti: Analytical Accounts of the Monetary Authorities, 1992-97

(In millions of Djibouti francs, end of period)

\begin{tabular}{|c|c|c|c|c|c|c|}
\hline & 1992 & 1993 & 1994 & 1995 & 1996 & 1997 \\
\hline Foreign assets & 14,823 & 13,347 & 13,962 & 12,688 & 13,682 & 12,052 \\
\hline Monetary cover $1 /$ & 11,814 & 10,859 & 11,277 & 10,089 & 10,112 & 9,977 \\
\hline Other foreign assets & 2,456 & 2,451 & 2,685 & 2,584 & 3,544 & 1,954 \\
\hline SDR holdings & 37 & 37 & -- & 15 & 26 & 137 \\
\hline Reserve position in the Fund & 516 & -- & -- & - & $\cdots$ & -16 \\
\hline Claims on government 2 / & -- & 307 & 534 & 534 & 1,275 & 1,839 \\
\hline Claims on commercial banks $2 /$ & 39 & 40 & 40 & 42 & 303 & 405 \\
\hline Reserve money & 12,187 & 11,250 & 11,869 & 10,370 & 10,250 & 10,144 \\
\hline Currency outside banks & 11,331 & 10,401 & 10,693 & 9,367 & 9,686 & 9,450 \\
\hline $\begin{array}{l}\text { Currency issued 3/ } \\
\text { Less: Currency held by }\end{array}$ & 11,814 & 10,859 & 11,277 & 10,089 & 10,112 & 9,977 \\
\hline treasury & 91 & 25 & 22 & 23 & 15 & 9 \\
\hline Less: Banks' cash & 392 & 433 & 562 & 699 & 411 & 518 \\
\hline Currency with banks & 392 & 433 & 562 & 699 & 411 & 518 \\
\hline Banks' deposits & 464 & 416 & 614 & 3.04 & 153 & 176 \\
\hline Foreign liabilities & 17 & 19 & 14 & 17 & 756 & 1,143 \\
\hline Government deposits $2 /$ & 956 & 719 & 743 & 603 & 943 & 515 \\
\hline Other items (net) & 1,702 & 1,706 & 1,910 & 2,274 & 3,311 & $-2,494$ \\
\hline
\end{tabular}

Source: Data provided by the Djibouti authorities.

1/ The monetary cover is equivalent to the sum of 'Currency outside banks', currency inside banks, and currency in the treasury.

2/ As of December 1996, includes overdraft advances.

$3 /$ Includes currency held by the treasury. 
Table 33. Djibouti: Analytical Accounts of the Commercial Banks, 1992-97

(In millions of Djibouti francs; end of period)

\begin{tabular}{|c|c|c|c|c|c|c|}
\hline & 1992 & 1993 & 1994 & 1995 & 1996 & 1997 \\
\hline Reserves & 868 & 925 & 1,148 & 1,065 & 638 & 778 \\
\hline Foreign assets & 35,561 & 38,967 & 37,594 & 37,314 & 30,801 & 29,719 \\
\hline Correspondent accounts & 34,088 & 35,925 & 36,135 & 35,685 & 28,982 & 28,619 \\
\hline Other & 1,473 & 3,042 & 1,459 & 1,629 & 1,819 & 1,100 \\
\hline Claims on the government & - & 446 & 2,525 & 2,144 & 1,569 & 1,678 \\
\hline Claims on the economy & 35,156 & 32,540 & 33,801 & 38,247 & 39,425 & 39,247 \\
\hline Claims on public enterprises & 444 & 483 & 419 & 464 & 599 & 778 \\
\hline Claims on the private sector & 34,712 & 32,057 & 33,382 & 37,783 & 38,826 & 38,469 \\
\hline Total assets/liabilities & 71,585 & 72,878 & 75,068 & 78,770 & 72,433 & 71,422 \\
\hline Demand deposits & 21,594 & 22,208 & 21,814 & 21,157 & 18,738 & 17,506 \\
\hline Time deposits & 22,986 & 22,094 & 23,030 & 26,841 & 23,185 & 23,943 \\
\hline Foreign liabilities & 15,009 & 15,073 & 15,755 & 16,190 & 15,819 & 14,810 \\
\hline Correspondent accounts & 9,125 & 7,181 & 8,792 & 8,452 & 7,106 & 7,691 \\
\hline Demand deposits & 3,933 & 4,291 & 3,748 & 3,402 & 4,270 & 2,886 \\
\hline Time deposits & 1,574 & 1,410 & 1,468 & 2,514 & 2,349 & 2,212 \\
\hline Other & 376 & 2,191 & 1,747 & 1,822 & 2,094 & 2,021 \\
\hline Government deposits & 929 & 925 & 2,089 & 727 & 568 & 605 \\
\hline Credit from the central bank & 39 & 40 & 40 & 40 & 40 & 406 \\
\hline Other items (net) & 11,029 & 12,540 & 12,340 & 13,815 & 14,083 & 14,152 \\
\hline
\end{tabular}

Source: Data provided by the Djibouti authorities. 
Table 34. Djibouti: Analytical Accounts of the Development Bank of Djibouti, 1992-97 (In millions of Djibouti francs: end of period)

\begin{tabular}{lrrrrrr}
\hline & 1992 & 1993 & 1994 & 1995 & 1996 & 1997 \\
\hline Cash and bank deposits & 251 & 261 & 167 & 80 & 30 & 24 \\
Claims on private sector & 4,202 & 4,472 & 4,277 & 4,126 & 3,537 & 2,530 \\
Claims on the government & 44 & 148 & 296 & 296 & 362 & 601 \\
Total assets/liabilities & 4,497 & 4,881 & 4,740 & 4,502 & 3,929 & 3,155 \\
Time deposits & 6 & 6 & 31 & 31 & 31 & 6 \\
Long-term foreign liabilities & 644 & 712 & 610 & 70 & 580 & 415 \\
Government lending funds & 1,999 & 1,788 & 1,542 & 1,533 & 1,180 & 919 \\
Capital accounts & 1,529 & 1,557 & 1,557 & 807 & 1,696 & 1,301 \\
Other items, net & 319 & 818 & 1,000 & 561 & 441 & 514 \\
\hline
\end{tabular}

Source: Data provided by the Djibouti authorities. 
Table 35. Djibouti: Structure of Interest Rates, 1995-97

(In percent per annum)

Time Deposits 1/

Min. Max
Lending Rate 2/

Min. Max

1995

3 rd quarter

4th quarter

1996

1st quarter

2nd quarter

3 rd quarter

4th quarter

1997

1st quarter

2nd quarter

3 rd quarter

4th quarter
$4.0-6.5$

9.9-12.8

$4.2-6.0$

9.4-13.1

$3.7-4.3$

9.2-13.9

$2.0-6.0$

9.2-13.6

$2.0-4.8$

9.5-13.2

2.1-4.0

9.5-13.3

Source: Data provided by the Djibouti authorities.

1/ The minimum balance for time deposits in Djibouti francs is DF $1,000,000$ with a minimum term of one month.

2/ Rate applicable to overdrafts of under DF 10 million. 
Table 36. Djibouti: Balance of Payments, 1992-97

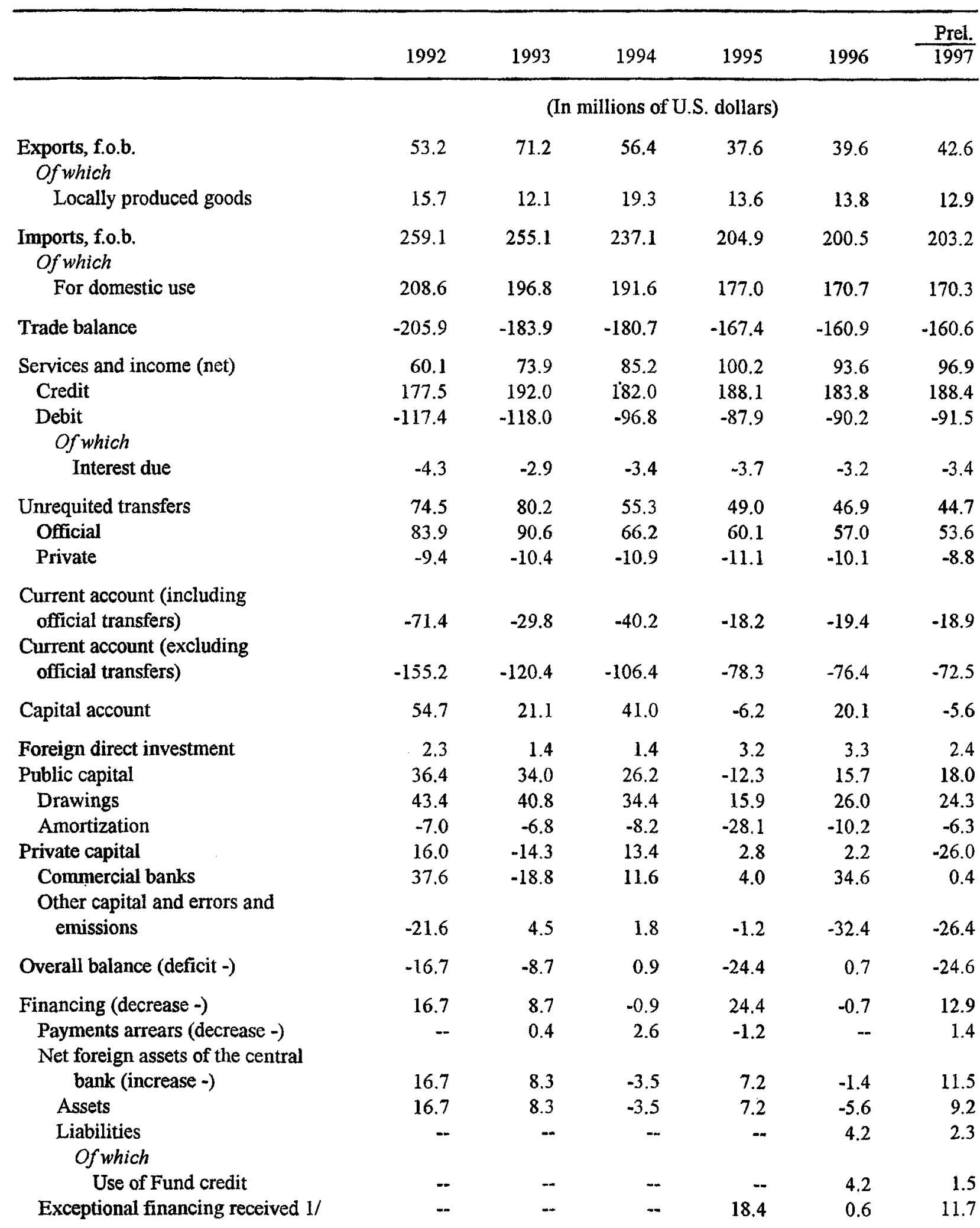


Table 36. Djibouti: Balance of Payments, 1992-97

\begin{tabular}{|c|c|c|c|c|c|c|}
\hline & 1992 & 1993 & 1994 & 1995 & 1996 & $\frac{\text { Prel. }}{1997}$ \\
\hline & \multicolumn{6}{|c|}{ (In percent of GDP) } \\
\hline Memorandum items: & & & & & & \\
\hline Imports for domestic use & 44.4 & 41.8 & 39.3 & 36.0 & 35.2 & 34.0 \\
\hline Exports of locally produced goods & 3.3 & 2.6 & 4.0 & 2.8 & 2.8 & 2.6 \\
\hline Imports by French army $2 /$ & 24.9 & 46.2 & 30.2 & 30.9 & 29.0 & 29.4 \\
\hline $\begin{array}{l}\text { Current account (including } \\
\text { official transfers) }\end{array}$ & -15.2 & -6.3 & -8.2 & -3.7 & -4.0 & -3.8 \\
\hline $\begin{array}{l}\text { Current account (excluding } \\
\text { official transfers) }\end{array}$ & -33.2 & -25.5 & -21.8 & -15.9 & -15.7 & -14.5 \\
\hline Outstanding debt & 45.0 & 52.8 & 57.0 & 54.1 & 58.6 & 60.9 \\
\hline $\begin{array}{l}\text { Debt service payments (in percent } \\
\text { of locally produced goods and } \\
\text { nonfactor services) }\end{array}$ & 6.4 & 5.6 & 6.6 & 7.7 & 7.7 & 5.5 \\
\hline $\begin{array}{l}\text { Debt stock (in percent of exports } \\
\text { of locally produced goods and } \\
\text { nonfactor services) }\end{array}$ & 129.2 & 143.2 & 156.3 & 150.3 & 163.0 & 172.2 \\
\hline Official reserves & & & & & & \\
\hline In millions of U.S. dollars & 83.4 & 75.1 & 78.6 & 71.4 & 77.0 & 67.8 \\
\hline In number of months of imports $3 /$ & 3.5 & 3.3 & 3.7 & 3.5 & 3.8 & 3.4 \\
\hline
\end{tabular}

Sources: Data provided by the Djibouti authorities; and Fund staff estimates.

1/ Includes debt relief and arrears regularization.

2/ This information is provided to facilitate comparison with pre-1996 reports, where imports by the French army were included as both imports and re-exports.

3/ Imports of goods and nonfactor services for domestic use. 
Table 37. Djibouti: Direction of Trade, 1992-97 1/

(In percent of total)

\begin{tabular}{|c|c|c|c|c|c|c|}
\hline & 1992 & 1993 & 1994 & 1995 & 1996 & $\frac{\text { Prel. }}{1997}$ \\
\hline \multicolumn{7}{|l|}{ I. Imports } \\
\hline Industrial countries & 53.8 & 50.4 & 47.1 & 43.2 & 44.7 & 47.8 \\
\hline France & 15.9 & 16.2 & 15.4 & 13.4 & 14.7 & 15.1 \\
\hline United Kingdom & 10.6 & 8.3 & 6.3 & 5.8 & 5.9 & 7.4 \\
\hline Italy & 6.2 & 6.4 & 6.6 & 5.9 & 7.5 & 6.5 \\
\hline Japan & 6.7 & 5.8 & 5.2 & 5.1 & 3.5 & 5.3 \\
\hline Netherlands & 3.6 & 4.0 & 4.1 & 3.8 & 4.0 & 3.9 \\
\hline Other countries & 10.7 & 9.6 & 9.5 & 9.4 & 9.1 & 9.6 \\
\hline Developing countries & 46.2 & 49.6 & 52.9 & 56.8 & 53.2 & 52.2 \\
\hline Africa & 5.3 & 6.6 & 9.1 & 10.1 & 13.1 & 14.3 \\
\hline Ethiopia & 4.1 & 5.4 & 7.6 & 8.2 & 10.7 & 9.5 \\
\hline Other countries & 1.1 & 1.2 & 1.6 & 1.9 & 2.4 & 4.7 \\
\hline Asia & 28.6 & 24.2 & 30.8 & 32.8 & 23.0 & 22.9 \\
\hline Taiwan Province of China & 2.8 & 2.8 & 2.5 & 4.2 & 5.6 & 3.6 \\
\hline Singapore & 2.7 & 2.6 & 3.1 & 4.5 & 3.7 & 3.3 \\
\hline Thailand & 7.1 & 9.5 & 15.8 & 15.8 & 3.7 & 5.4 \\
\hline Hong Kong, China & 3.0 & 3.0 & 2.5 & 2.0 & 1.1 & 2.3 \\
\hline India & 6.1 & 5.6 & 3.5 & 3.0 & 1.6 & 4.0 \\
\hline Other countries & 6.9 & 0.7 & 3.3 & 3.1 & 7.2 & 4.2 \\
\hline Middle East & 11.0 & 17.4 & 10.9 & 11.3 & 15.0 & 13.1 \\
\hline Bahrain & 1.3 & 1.7 & 2.4 & 2.6 & 3.5 & 2.3 \\
\hline Saudi Arabia & 6.6 & 11.5 & 6.1 & 6.1 & 7.5 & 7.6 \\
\hline Other & 3.1 & 4.2 & 2.4 & 2.6 & 4.0 & 3.2 \\
\hline Other countries & 1.2 & 1.4 & 2.0 & 2.6 & 2.1 & 1.9 \\
\hline Total & 100.0 & 100.0 & 100.0 & 100.0 & 100.0 & 100.0 \\
\hline \multicolumn{7}{|l|}{ II. Exports } \\
\hline Industrial countries & 4.5 & 3.1 & 3.0 & 8.1 & 3.0 & 3.1 \\
\hline Developing countries & 95.5 & 96.9 & 97.0 & 91.9 & 96.3 & 96.9 \\
\hline Africa & 63.7 & 52.2 & 57.0 & 76.7 & 72.6 & 74.3 \\
\hline Somalia & 29.0 & 23.7 & 26.2 & 35.3 & 39.3 & 36.9 \\
\hline Ethiopia & 34.0 & 27.8 & 30.7 & 41.4 & 33.3 & 37.4 \\
\hline Middle East & 28.1 & 42.7 & 38.6 & 11.7 & 23.7 & 22.6 \\
\hline Yemen, Republic of & 25.4 & 40.7 & 37.3 & 7.6 & 20.7 & 19.0 \\
\hline Saudi Arabia & 2.2 & 1.6 & 0.5 & 3.2 & 3.0 & 2.1 \\
\hline Other & 3.7 & 2.0 & 1.4 & 3.4 & 0.7 & 1.5 \\
\hline Total & 100.0 & 100.0 & 100.0 & 100.0 & 100.0 & 100.0 \\
\hline
\end{tabular}

Source: IMF, Direction of Trade Statistics.

$1 /$ Based on data of trading partner countries. 
Table 38. Djibouti: Domestically Consumed Imports Classified by Main Product Groups, 1992-97

(In millions of Djibouti francs)

\begin{tabular}{|c|c|c|c|c|c|c|}
\hline & & & & & & Prel. Est. \\
\hline & 1992 & 1993 & 1994 & 1995 & 1996 & 1997 \\
\hline Food and beverages & 10,408 & 9,824 & 9,562 & 8,833 & 8,519 & 8,718 \\
\hline Tobacco & 568 & 536 & 522 & 482 & 465 & 476 \\
\hline Khat & 4,346 & 4,102 & 3,993 & 3,689 & 3,557 & 3,641 \\
\hline Minerals & 490 & 463 & 450 & 416 & 401 & 410 \\
\hline Petroleum products & 2,973 & 2,806 & 2,731 & 2,523 & 2,433 & 2,490 \\
\hline Chemical products & 1,773 & 1,674 & 1,629 & 1,505 & 1,451 & 1,485 \\
\hline Plastic products & 555 & 524 & 510 & 471 & 455 & 465 \\
\hline Paper products, newspapers, books & 714 & 674 & 656 & 606 & 585 & 598 \\
\hline Clothing and footwear & 2,808 & 2,651 & 2,580 & 2,383 & 2,299 & 2,353 \\
\hline Stone, cement, ceramic, and glass products & 357 & 337 & 328 & 303 & 292 & 299 \\
\hline Wood, wood products, and furniture & 719 & 678 & 660 & 610 & 588 & 602 \\
\hline Metals and metal products & 1,787 & 1,686 & 1,641 & 1,516 & 1,462 & 1,497 \\
\hline Machinery and electric appliances & 7,051 & 6,655 & 6,478 & 5,984 & 5,771 & 5,906 \\
\hline Vehicles and transport equipment & 1,254 & 1,183 & 1,152 & 1,064 & 1,026 & 1,050 \\
\hline Other products & 248 & 234 & 228 & 210 & 203 & 208 \\
\hline Other transactions & 1,014 & 957 & 931 & 860 & 830 & 849 \\
\hline Total & 37,065 & 34,984 & 34,051 & 31,457 & 30,337 & 31,048 \\
\hline
\end{tabular}

Sources: Data provided by the Djibouti authorities; and Fund staff estimates. 
Table 39. Djibouti: External Public- and Publicly-Guaranteed Debt, External Debt Service, 1993-97

(In millions of U.S. dollars)

\begin{tabular}{|c|c|c|c|c|c|}
\hline & 1993 & 1994 & 1995 & 1996 & $\frac{\text { Prel. }}{1997}$ \\
\hline \multicolumn{6}{|l|}{ Medium- and long-term debt and } \\
\hline $\begin{array}{l}\text { Bilateral loans } \\
\text { Of which }\end{array}$ & \multicolumn{4}{|c|}{ Of which } & 98.6 \\
\hline China & 4.2 & 4.2 & 1.9 & 0.0 & 9.8 \\
\hline France & 9.2 & 9.2 & 9.1 & 9.8 & 1.0 \\
\hline Italy & 29.6 & 35.3 & 35.9 & 45.8 & 45.8 \\
\hline Kuwait & 23.8 & 27.4 & 27.1 & 28.0 & 29.7 \\
\hline Saudi Arabia & 12.1 & 14.4 & 8.1 & 12.9 & 12.3 \\
\hline United Arab Emirates & 3.7 & 4.1 & 0.0 & 0.0 & 0.0 \\
\hline $\begin{array}{l}\text { Multilateral loans } \\
\text { Of which }\end{array}$ & 166.3 & 183.1 & 183.2 & 187.9 & 205.9 \\
\hline $\begin{array}{l}\text { African Development Fund } \\
\text { Arab Fund for Economic and Social }\end{array}$ & 52.8 & 57.9 & 60.4 & 66.0 & 58.3 \\
\hline Development & 15.0 & 14.5 & 14.3 & 13.3 & 13.9 \\
\hline Arab monetary Fund & 0.0 & 0.0 & 0.0 & 0.0 & 0.8 \\
\hline European Development & 2.5 & 3.2 & 3.1 & 3.6 & 3.6 \\
\hline International Development Association & 39.6 & 41.1 & 43.8 & 43.7 & 41.1 \\
\hline \multicolumn{6}{|l|}{ International Fund for Agriculture } \\
\hline Development & 2.0 & 1.9 & 1.8 & 2.1 & 3.5 \\
\hline Islamic Development Bank & 2.8 & 2.5 & 2.4 & 2.8 & 8.5 \\
\hline OPEC Special Fund & 1.9 & 1.7 & 1.6 & 1.9 & 4.4 \\
\hline Disbursement & 40.8 & 34.5 & 15.9 & 30.2 & 26.5 \\
\hline Amortization & 6.8 & 8.3 & 28.1 & 10.2 & 6.3 \\
\hline Interest payment & 3.8 & 4.3 & 4.6 & 4.1 & 4.4 \\
\hline Short term & 0.9 & 0.9 & 0.9 & 0.9 & 1.0 \\
\hline Long term & 2.9 & 3.4 & 3.7 & 3.2 & 3.4 \\
\hline Arrears, stock end of period & 0.4 & 3.0 & 1.8 & 2.7 & 2.4 \\
\hline Stock beginning of period & .. & 0.4 & 3.0 & 1.8 & 2.7 \\
\hline New accumulation & -- & 2.6 & 0.3 & 0.9 & 0.8 \\
\hline Settlement & - & 0.0 & -1.5 & 0.0 & -1.2 \\
\hline \multicolumn{6}{|l|}{ Memorandum items: } \\
\hline External public debt as percent of GDP & 52.8 & 57.0 & 54.1 & 58.6 & 60.9 \\
\hline \multicolumn{6}{|l|}{ Total debt in percent of export } \\
\hline and nonfactor services & 143.2 & 156.3 & 150.3 & 163.0 & 172.2 \\
\hline \multicolumn{6}{|l|}{$\begin{array}{l}\text { Total debt service in percent of } \\
\text { export of locally produced goods }\end{array}$} \\
\hline and nonfactor services & 5.6 & 6.6 & 7.7 & 7.7 & 5.5 \\
\hline \multicolumn{6}{|l|}{ Average interest rate on total } \\
\hline debt (in percent) & 1.42 & 1.45 & 1.64 & 1.36 & 1.38 \\
\hline \multicolumn{6}{|l|}{ Average interest rate on long-term } \\
\hline debt (in percent) & 1.16 & 1.22 & 1.41 & 1.13 & 1.13 \\
\hline
\end{tabular}

Sources: Data provided by Djibouti authorities; the World Bank Debtor Reporting System; and Fund Staff estimates.

1/ Refers to the debt of both the central government and the public enterprise sector. 
Table 40. Djibouti: Exchange Rate Data, Period Averages, 1992-97

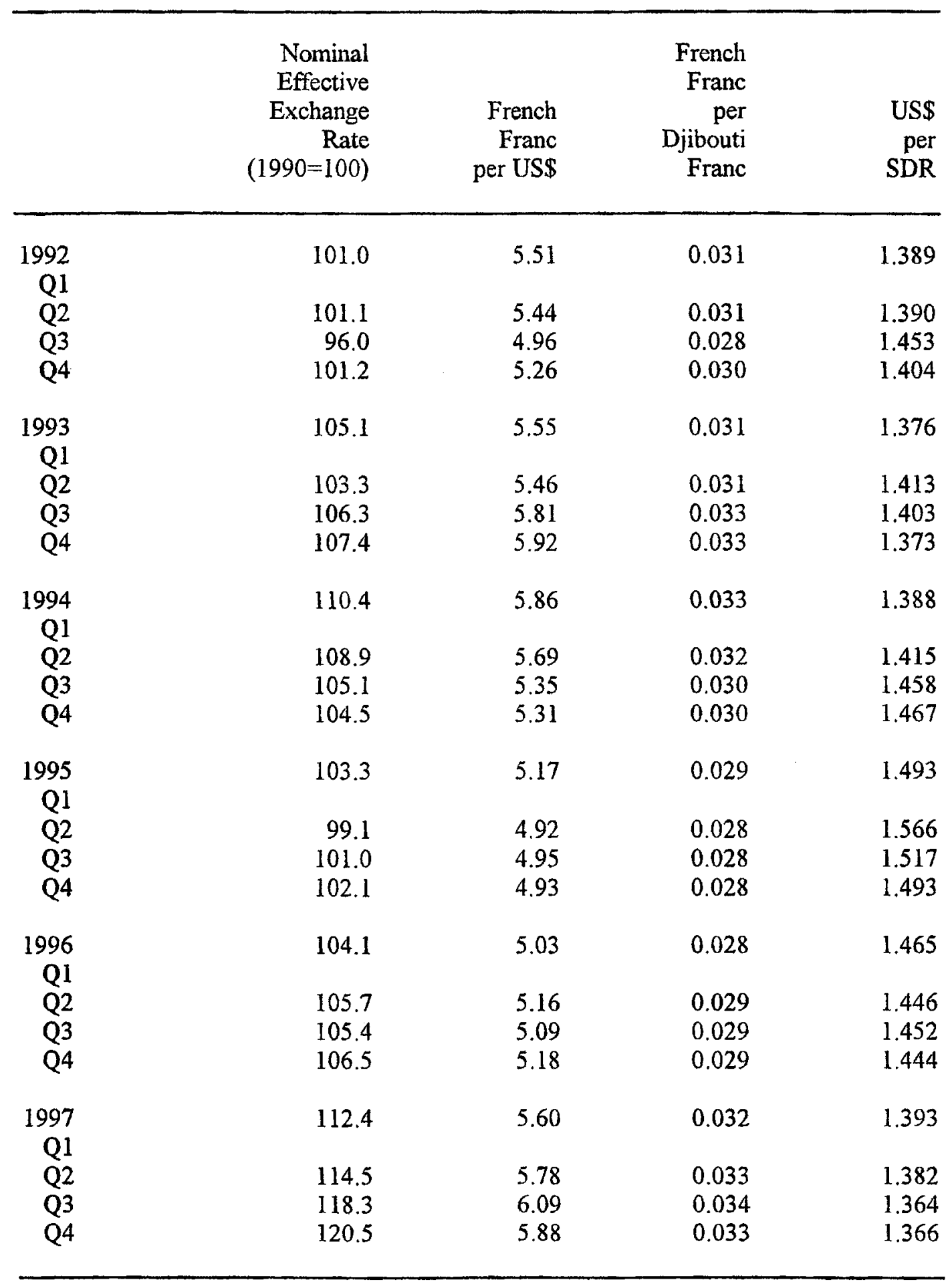

Sources: IMF, Information Notice System; and International Financial Statistics. 
Djibouti: Summary of the Tax System

(as of March 31, 1998)

$\operatorname{Tax}$

Nature of Tax

Exemptions and Deductions

Assessment Base and Rates

\section{Taxes on Income, Profits, and Capital Gains}

\subsection{Individual}

- Tax on wages and salaries

\section{- Patriotic contribution'}

\subsection{Corporate}

- Business lump-sum tax

A progressive monthly tax levied on all wages, benefits, in cash and in kind, and pensions paid in Dijbouti. The tax, payable by the employee, is withheld from wages and salaries

Exemptions:

Monthly wages below DF 25,000

Certain allowances and reim-

bursements of actual occupational expenses

A flat monthly tax levied on all Djibouti wages and salaries

Levied on preceding year's turnover of all natural or legal persons exercising

handicraft, commercial, or industrial activities, or liberal professions

Monthly cash income rate:

DF 25,000-30,000:

DF 30,000-100,000:

DF $100,000-200,000$ :

DF 200,000-400,000:

DF 400,000-600,000:

DF 600,000-800,000:

over DF 800,000

\section{$2 \%$}

$6 \%$

$10 \%$

$14 \%$

$19 \%$

$25 \%$

$32 \%$

Monthly in-kind income rate:

less than DF 30,000 :

DF $30,000-100,000$ :

$0 \%$

DF 100,000-150,000:

DF 150,000-250,000:

DF 250,000-350,000:

DF 350,000-450,000:

DF 450,000-550,000:

over DF 550,000

Total of wages, benefits, and pensions less than DF 60,000 per month

Not deductible from taxable income

1 percent of turnover but not less than DF 120,000

10 percent of taxable income 
Djibouti: Summary of the Tax System

(as of March 31, 1998)

\begin{tabular}{|c|c|c|}
\hline $\operatorname{Tax}$ & Nature of Tax & Exemptions and Deductions \\
\hline $\begin{array}{l}\text { - Tax on professional } \\
\text { earnings }\end{array}$ & $\begin{array}{l}\text { Levied by self-assessment on profits and } \\
\text { similar income of members of the } \\
\text { professions and on the remuneration of } \\
\text { business operators with a controlling } \\
\text { interest in their companies if the amount } \\
\text { of the tax is higher than that on the } \\
\text { business lump sum tax }\end{array}$ & $\begin{array}{l}\text { Exemptions: } \\
\text { Net profits of less than DF } 600,000 \text { a } \\
\text { year }\end{array}$ \\
\hline $\begin{array}{l}\text { - Tax on business profits of } \\
\text { individuals }\end{array}$ & $\begin{array}{l}\text { Levied by self-assessment on the profits } \\
\text { of unincorporated industrial, commercial, } \\
\text { or craft enterprises }\end{array}$ & $\begin{array}{l}\text { Exemptions: } \\
\text { Investment income } \\
\text { As provided for in Investment Code } \\
\text { Income from real estate }\end{array}$ \\
\hline \multirow[t]{2}{*}{ - Tax on company profits } & $\begin{array}{l}\text { Levied by self-assessment on the profits } \\
\text { of joint-stock companies, cooperatives, } \\
\text { and partnerships (including government- } \\
\text { owned enterprises, mixed-economy } \\
\text { enterprises, and industrial or commercial } \\
\text { public enterprises) }\end{array}$ & $\begin{array}{l}\text { Exemptions: } \\
\text { Income from real estate } \\
\text { As provided for in Investment Code } \\
\text { Net profits of less than DF } 600,000 \text { a } \\
\text { year }\end{array}$ \\
\hline & $\begin{array}{l}\text { The following three categories of } \\
\text { taxpayers are subject to a system of } \\
\text { estimated payments pending determina- } \\
\text { tion of their assessable profits for } \\
\text { purposes of the income and profits tax } \\
\text { (see 1.3): } \\
\text { Itinerant enterprises; } \\
\text { Livestock importers; and } \\
\text { Khat importers }\end{array}$ & \\
\hline
\end{tabular}

1.3 Other Unallocated Taxes on Income, Profits, and Capital Gains

25 percent of net taxable annual profits

25 percent of net taxable annual profits

Charchari:

10 percent of $c$.i.f. price of imported merchandise plus indirect duties

Livestock importers:

DF 800 per sheep or goat

DF 1,000 per head of cattle

Khat importers:

DF 100 per kilogram of khat 
Djibouti: Summary of the Tax System

(as of March 31, 1998)

$\frac{\operatorname{Tax}}{-\begin{array}{l}\text { Tax on real estate capital } \\ \text { gains }\end{array}}$

2. Taxes on Property
- Real property tax on unbuilt land
Assessed on the annual rental value of the property beginning with the sixth year following the year of building completion. Buildings may be exempted for a maximum of ten years after completion under the Investment Code

Levied on unbuilt land, on land under buildings temporarily exempt from the real property tax on buildings during the exemption period, and on vacant land belonging to a building to the extent that it exceeds three times the built-on area
Exemptions and Deductions

Assessment Base and Rates evied on buildings upon transfer and payable by transferrer. Same for land without buildings

Exemptions:

Principal residences

Real estate assets of enterprises

Sales below DF 100,000

\section{5 percent of net capital gain}

Exemptions:

Public buildings

Religious and sports facilities

Industrial facilities (Investment Code)

Deductions:

20 percent of rental value for amortization, maintenance, and insurance

Exemptions:

Government property

Land used for schools, sports, humanitarian, or social welfare purposes

Agricultural land in farming use outside urban areas. Land owed by international organizations, embassies, and consulates of accredited countries

\section{Exemptions:}

Government property

Properties used for educational,

sporting, hume

purposes
DF 0-1,120,00:

DF $1,120,001-3,840,000$ : $20 \%$

DF 3,840,001-6,720,000: $25 \%$

Over DF $6,720,000$ : $\quad 30 \%$ 
Djibouti: Summary of the Tax System

(as of March 31, 1998)

\begin{tabular}{c}
\hline Tax \\
\hline property \\
Tax on undeveloped real \\
- Garbage removal tax
\end{tabular}

Levied on real property not recorded as being in use by January 1 of the tax year, or no longer in use for a period of over five years

Levied yearly from the owners, users, or principal tenants of real property on the same basis as the real property tax on buildings

Payable on documents under private seal, public civil documents, judicial documents, verbal leases, and transfers inter vivos or causa mortis

Prorated duty applies to all legal

documents involving obligation, release, condemnation, placement or payment of money or securities, transfers of real and movable property, and insurance premiums

A fixed duty is collected on all other legal documents

A progressive duty is payable on

transfers inter vivos or causa mortis
Developed agricultural land

Properties of international

organizations, embassies, and

consulates

\section{Exemptions:}

Public property

Agricultural plots

Lots belonging to international

organizations, embassies, and

consulates of accredited countries

\section{Exemptions:}

Industrial enterprises

Buildings in governmental use

Buildings without garbage removal

services

4.5 percent of rental value after

20 percent deduction

\section{Exemptions:}

Transactions to which the government

is a party

Investment Code approvals

Miscellaneous (see Registration and

Stamp Duty Code)

Embassies

5 percent of assessed value

Prorated duty:

5 percent and 10 percent

Fixed duty:

DF 2,000

DF 4,000

DF 6,000

Progressive duty:

See Registration Code schedule 
(as of March 31, 1998)

- Recordation duty

3. Domestic Taxes on Goods and Services

3.1 Taxes on Specific Services

- Tax on construction permits

- Gambling levy

3.2 Taxes on Use of Goods or on Permission to Use Goods or to Perform Activities

- Patent (business license duty)
Levied on the provisional or definitive grant or the conveyance of real property, and for the registration or cancellation of mortgages. Subrogation of mortgages is not subject to the duty. Definitive grants are subject to prior assessment

\section{Exemptions:}

The government

Investment Code approvals
Recordation:

DF $200,500,1,000$ or 5,000 per sheet or act
Levied upon filing of applications for construction permits with the Ministry of Public Works, Town Planning, and Housing

Payable by all persons engaged in an industrial or commercial activity within Djibouti. A special annual patent is levied on industrial, commercial, or agricultural companies head-quartered in Djibouti but operating mainly abroad. The same applies to holding companies and financing companies headquartered in Djibouti. For collection purposes, a distinction is made between the regular patent and the prepaid patent
Exemptions:

Government buildings

Investment Code approvals
Exemptions:

The government and public enterprises

Certain occupations (e.g., midwives, fishermen)

As provided for in the Investment Code; the term and conditions of the exemption are set forth in the approval decision

Importer patents are not required for transshipment, transit, provisioning, and fueling of ships and aircraft or for re-export
1.5 percent of planned construction cost

30 percent of the gross proceeds from gambling

The patent is made up of a fixed duty and a prorated one, both specified for each industry in the general schedule of patents

Fixed duty: Set for each enterprise carrying out operations subject to the patent. Persons engaged in various kinds of business through a single enterprise pay the patent only on the operations subject to the highest duty, except where the operations are defined as not consistent for purposes of taxation 
(as of March 31, 1998)

The official assessment for the latter is used only to finalize the amount; they apply mainly to business of an itinerant nature (e.g., traveling salesmen).

(Excludes the surcharge of 7.5 percent

levied for the Intemational Chamber of

Commerce and Industry)

- License fees

- Market tax

- Weights and measures duty

- Drivers' license and registration fee
Payable for the entire calendar year by all persons authorized to sell beverages. The amount depends on the type of

establishment and on its hours and location

For permits to sell at retail in a market

Annual duty levied by the Economic Affairs and Prices Service

Specific fees apply to the issue of drivers' licenses, registrations, and transfer authorizations (non cumulables (NC)); (e.g., an importer's patent: $\mathrm{NC}$ )

Prorated duty: Based on the rental value of the premises, whatever their type, in which the occupations subject to the duty are performed

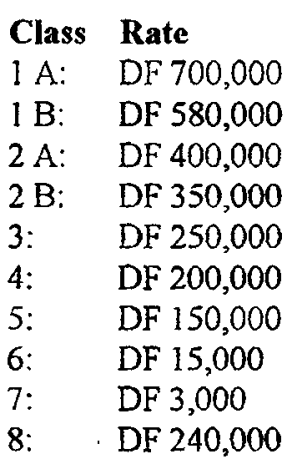

Covered market: DF 10 per day Covered stalls: DF 210 per month Pavilion:

Full place-DF 2,000 per month Half place-DF 1,000 per month

DF 100-5,000 per weighing or measuring device

Driver's license:

Issue: DF 7,000

Change:

Duplicate:

Registration

DF 4,500 per HP (DF 90,000

maximum) 
Djibouti: Summary of the Tax System

(as of March 31, 1998)

\begin{tabular}{|c|c|}
\hline $\operatorname{Tax}$ & Nature of Tax \\
\hline $\begin{array}{l}\text { - Differentiated motor } \\
\text { vehicle fee }\end{array}$ & Levied on all motor vehicles \\
\hline $\begin{array}{l}\text { - Recreational boat } \\
\text { registration fee }\end{array}$ & $\begin{array}{l}\text { Levied annually on boat owners in } \\
\text { accordance with engine size }\end{array}$ \\
\hline \multirow[t]{2}{*}{$\begin{array}{l}\text { 3.3 Domestic consumption tax } \\
\text { (TIC) }\end{array}$} & $\begin{array}{l}\text { Levied on all goods imported into and } \\
\text { consumed in Djibouti }\end{array}$ \\
\hline & $\begin{array}{l}\text { Vegetable oil, sugar, food paste, powder } \\
\text { milk, and medicines }\end{array}$ \\
\hline $\begin{array}{l}\text { - Dairy products and fruit } \\
\text { juice tax }\end{array}$ & $\begin{array}{l}\text { Levied on imports of: } \\
\text { Dairy products } \\
\text { Fruit juices } \\
\text { Other carbonated flavored beverages }\end{array}$ \\
\hline - Meat inspection tax & $\begin{array}{l}\text { Levied for inspection of meat and other } \\
\text { imported foodstuffs of animal origin }\end{array}$ \\
\hline \multicolumn{2}{|l|}{ - Toiletries } \\
\hline \multicolumn{2}{|l|}{ - Perfumes } \\
\hline $\begin{array}{l}\text { - Mineral water tax and soft } \\
\text { drinks }\end{array}$ & $\begin{array}{l}\text { Levied on imported mineral water and } \\
\text { locally-produced soft drinks }\end{array}$ \\
\hline
\end{tabular}

Exemptions and Deductions

Exemptions:

Professional fishermen

\section{Exemptions:}

Diplomatic privileges

Investment Code approval

Special agreements

Specific decrees or arrêtés

Vegetable oil, sugar, food paste, powder

Other carbonated flavored beverages

evied for inspection of meat and other locally-produced soft drinks
Assessment Base and Rates

$\begin{array}{ll}1-4 \mathrm{HP} & \text { DF } 15,000 \\ 5-9 \mathrm{HP} & \text { DF } 20,000 \\ 10-20 \mathrm{HP} & \text { DF } 25,000 \\ \text { Over } 20 \mathrm{HP} & \text { DF } 30,000\end{array}$

DF 500 per actual HP

Base: c.i.f. value or reference value 5 percent on food products

Specific duty of DF $5 / \mathrm{kg}$ on fresh vegetables and cereals

Standard rates (on valeurs mercuriales):

10 percent

20 percent

33 percent

Rate: 10 percent

DF 160 per kilogram (net)

Meat, fish, cheese, yogurt, honey: DF 100 per kilogram

Milk, eggs: DF 20 per kilogram

DF 300 per liter of alcohol content

DF 2,500 per liter of alcohol content

DF 14 per liter 
Djibouti: Summary of the Tax System

(as of March 31, 1998)

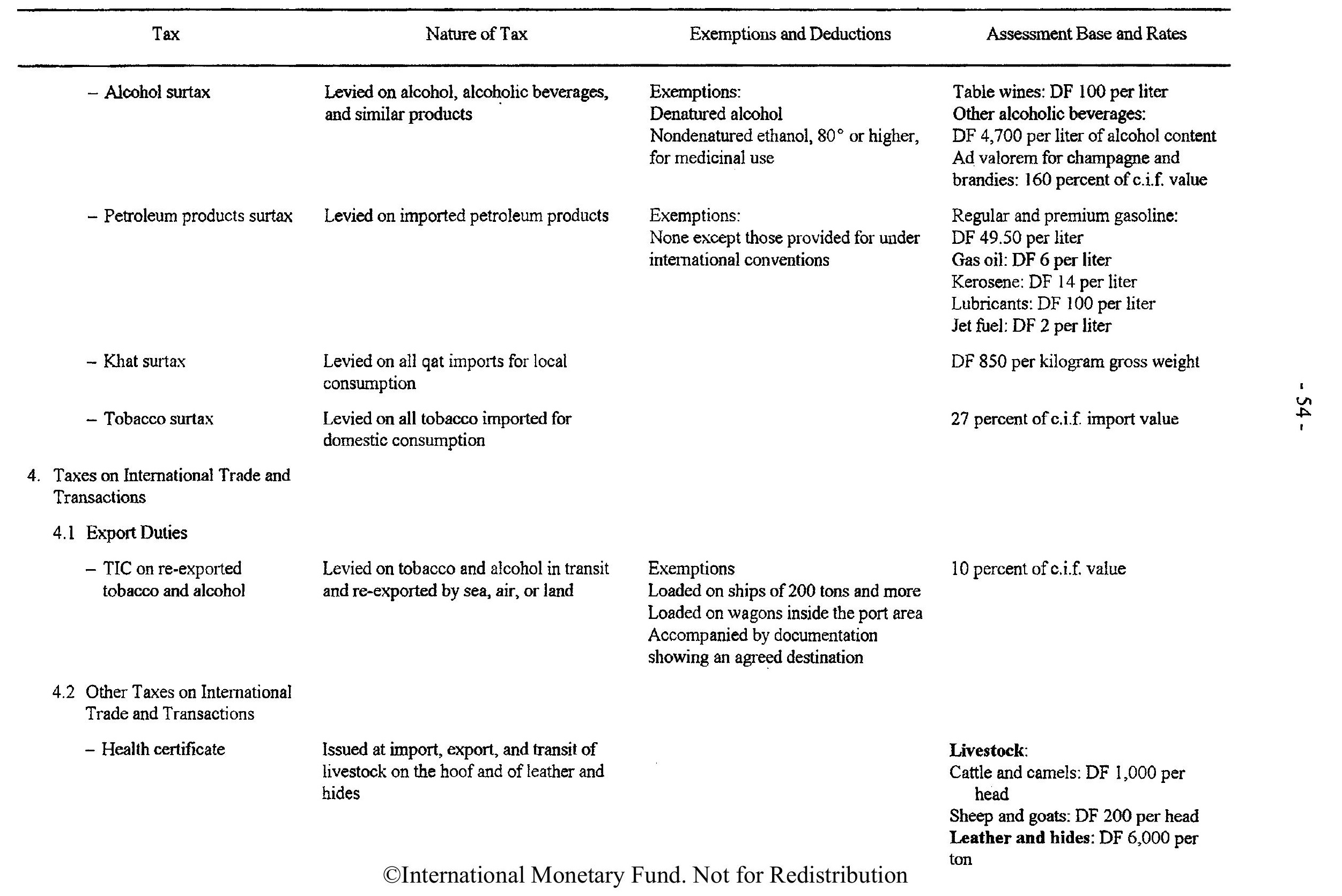


Djibouti: Summary of the Tax System

(as of March 31, 1998)

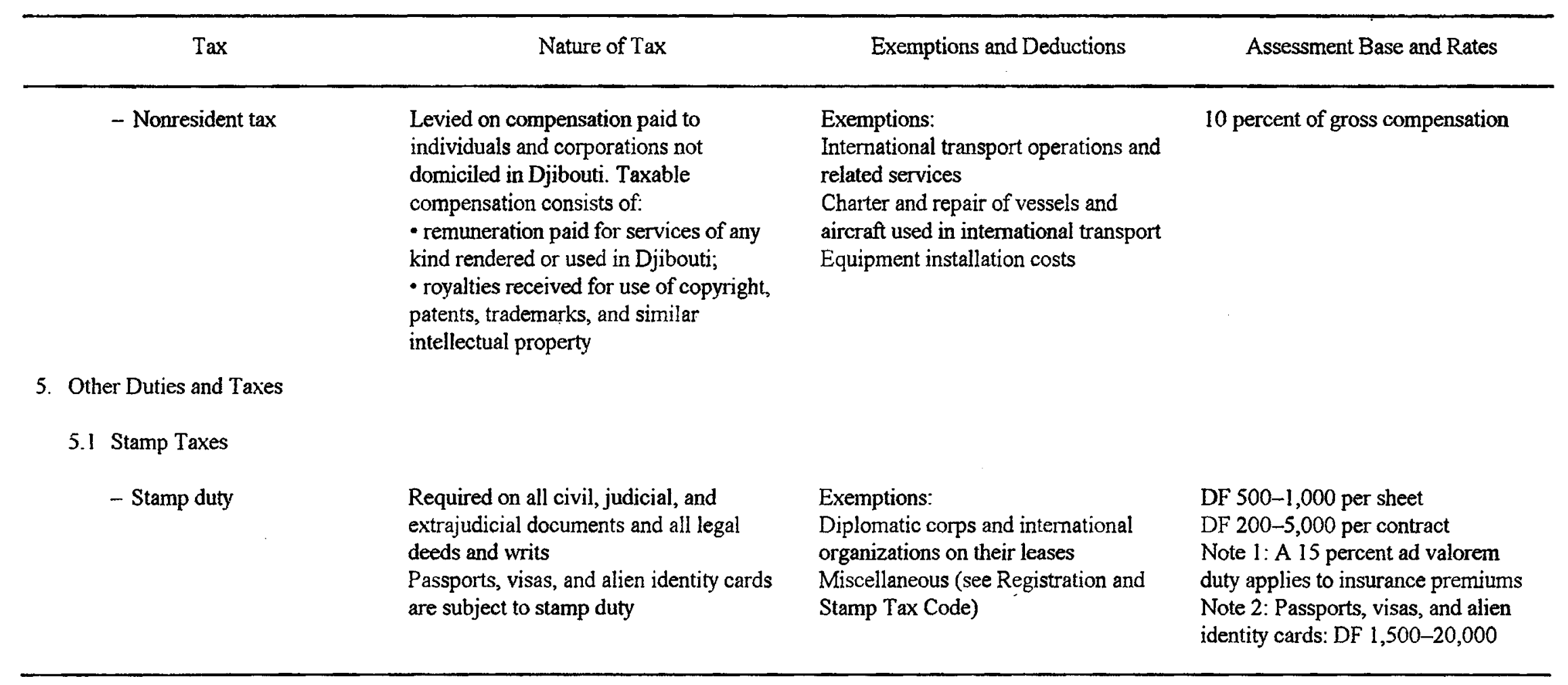

Source: Information provided by the Djibouti authorities.

${ }^{1}$ A temporary tax introduced in 1992 to finance exceptional expenditures associated with the general mobilization. It currently applies to all wage earners. For government and public enterprise employees, it is called budget withholding. 


\section{Djibouti-Public Agencies and Enterprises}

\section{Social security agencies}

Caisse Nationale de Retraite, CNR, Ministry of Finance Caisse des Prestations Sociales, CPS, Ministry of Labor Coopérative Militaire de Retraite, Ministry of Defense

Service Medical Inter-entreprises, SMI, Ministry of Labor

\section{Public agencies}

Institut Scientifique d'Etudes et de Recherches, ISERST, Presidency Palais du Peuple, Presidency

Chambre de Commerce International de Djibouti, CCID, Ministry of Commerce Office National d'Approvisionnement et de Commercialisation, ONAC, Ministry of Commerce, Transport and Tourism

Etablissement Public des Hydrocarbures, EPH, Ministry of Commerce Office National des Refugiés et Sinistrés (ONARS) - Presidency Central Regional Inter-Linguistique (CRIL) - Ministry of Education Office National de Tourisme et l'Artisanat

\section{Nonfinancial public enterprises}

Electricité de Djibouti, EdD, Ministry of Industry Office National des Eaux de Djibouti, ONED, Ministry of Industry Aéroport International de Djibouti, AID, Ministry of Commerce Port Autonome International de Djibouti, PAID, Ministry of Transport and Telecommunications

Office des Postes et Télégraphes, OPT, Ministry of Interior Société des Télécommunications Internationales, STID, Ministry of Interior Chemin de Fer Djibouti-Ethiopien, CDE, Ministry of Commerce, and the Government of Ethiopia

Société Hotelière de l'Etat, Ministry of Commerce Société des Eaux de Tadjourah, Ministry of Industry

Société Immobilière de Djibouti, SID, Presidency and Ministry of Public Works Laiterie de Djibouti, LDJ, Ministry of Industry Magasins Généraux, Chamber of Commerce Société Aliments Bétail, (SAB), Ministry of Agriculture

Financial public enterprises

Banque Nationale de Djibouti, BND

Banque de Développement de Djibouti, BDD 